Groups Geom. Dyn. 4 (2010), 873-900

DOI $10.4171 / \mathrm{GGD} / 112$
Groups, Geometry, and Dynamics

(C) European Mathematical Society

\title{
Nil graded self-similar algebras
}

\author{
Victor M. Petrogradsky ${ }^{1}$, Ivan P. Shestakov ${ }^{2}$ and Efim Zelmanov ${ }^{3}$
}

Abstract. In [19], [24] we introduced a family of self-similar nil Lie algebras $\boldsymbol{L}$ over fields of prime characteristic $p>0$ whose properties resemble those of Grigorchuk and Gupta-Sidki groups. The Lie algebra $\boldsymbol{L}$ is generated by two derivations

$$
\begin{aligned}
& v_{1}=\partial_{1}+t_{0}^{p-1}\left(\partial_{2}+t_{1}^{p-1}\left(\partial_{3}+t_{2}^{p-1}\left(\partial_{4}+t_{3}^{p-1}\left(\partial_{5}+t_{4}^{p-1}\left(\partial_{6}+\cdots\right)\right)\right)\right)\right), \\
& v_{2}=\partial_{2}+t_{1}^{p-1}\left(\partial_{3}+t_{2}^{p-1}\left(\partial_{4}+t_{3}^{p-1}\left(\partial_{5}+t_{4}^{p-1}\left(\partial_{6}+\cdots\right)\right)\right)\right)
\end{aligned}
$$

of the truncated polynomial ring $K\left[t_{i}, i \in \mathbb{N} \mid t_{i}^{p}=0, i \in \mathbb{N}\right]$ in countably many variables. The associative algebra $\boldsymbol{A}$ generated by $v_{1}, v_{2}$ is equipped with a natural $\mathbb{Z} \oplus \mathbb{Z}$-gradation. In this paper we show that for $p$, which is not representable as $p=m^{2}+m+1, m \in \mathbb{Z}$, the algebra $\boldsymbol{A}$ is graded nil and can be represented as a sum of two locally nilpotent subalgebras. L. Bartholdi [3] and Ya. S. Krylyuk [15] proved that for $p=m^{2}+m+1$ the algebra $A$ is not graded nil. However, we show that the second family of self-similar Lie algebras introduced in [24] and their associative hulls are always $\mathbb{Z}^{p}$-graded, graded nil, and are sums of two locally nilpotent subalgebras.

Mathematics Subject Classification (2010). 17B05, 17B50, 17B66, 17B65, 16P90, 11B39.

Keywords. Modular Lie algebras, growth, nil-algebras, self-similar, Gelfand-Kirillov dimension, Lie algebras of vector fields, Grigorchuk group, Gupta-Sidki group.

\section{Definitions and constructions}

Let $L$ be a Lie algebra over a field $K$ of characteristic $p>0$ and let ad $x: L \rightarrow L$, ad $x(y)=[x, y]$ for $x, y \in L$, be the adjoint map. Recall that $L$ is called a restricted Lie algebra or Lie p-algebra [12], [26], [1] if L additionally affords a unary operation $x \mapsto x^{[p]}, x \in L$, satisfying

i) $(\lambda x)^{[p]}=\lambda^{p} x^{[p]}$ for all $\lambda \in K, x \in L$;

ii) $\operatorname{ad}\left(x^{[p]}\right)=(\operatorname{ad} x)^{p}$ for all $x \in L$;

\footnotetext{
${ }^{1}$ The first author was partially supported by grants FAPESP 05/58376-0 and RFBR-07-01-00080.

${ }^{2}$ The second author was partially supported by grants FAPESP 05/60337-2 and CNPq 304991/2006-6.

${ }^{3}$ The third author was partially supported by the NSF grant DMS- 0758487.
} 
iii) for all $x, y \in L$ one has

$$
(x+y)^{[p]}=x^{[p]}+y^{[p]}+\sum_{i=1}^{p-1} s_{i}(x, y),
$$

where $i s_{i}(x, y)$ is the coefficient of $Z^{i-1}$ in the polynomial $(\operatorname{ad}(Z x+y))^{p-1}(x)$ in $L[Z]$, with $Z$ is an indeterminate. Also, $s_{i}(x, y)$ is a Lie polynomial in $x, y$ of degrees $i$ and $p-i$, respectively.

Suppose that $L$ is a restricted Lie algebra and $X \subset L$. Then by $\operatorname{Lie}_{p}(X)$ we denote the restricted subalgebra generated by $X$. Let $H \subset L$ be a Lie subalgebra, i.e., $H$ is a vector subspace which is closed under the Lie bracket. Then by $H_{p}$ we denote the restricted subalgebra generated by $H$. In what follows by an associative enveloping algebra of a Lie algebra we mean the associative algebra without 1 .

We recall the notion of growth. Let $A$ be an associative (or Lie) algebra generated by a finite set $X$. Denote by $A^{(X, n)}$ the subspace of $A$ spanned by all monomials in $X$ of length not exceeding $n$. If $A$ is a restricted Lie algebra, then we define [16] $A^{(X, n)}=\left\langle\left[x_{i_{1}}, \ldots, x_{i_{s}}\right]^{p^{k}} \mid x_{i_{j}} \in X, s p^{k} \leq n\right\rangle_{K}$. In either situation, one defines the growth function:

$$
\gamma_{A}(n)=\gamma_{A}(X, n)=\operatorname{dim}_{K} A^{(X, n)}, \quad n \in \mathbb{N} .
$$

The growth function clearly depends on the choice of the generating set $X$. Furthermore, it is easy to see that the exponential growth is the highest possible growth for Lie and associative algebras. The growth function $\gamma_{A}(n)$ is compared with the polynomial functions $n^{k}, k \in \mathbb{R}^{+}$, by computing the upper and lower Gelfand-Kirillov dimensions [14], namely

$$
\begin{aligned}
\operatorname{GKdim} A & =\varlimsup_{n \rightarrow \infty} \frac{\ln \gamma_{A}(n)}{\ln n}, \\
\underline{\operatorname{GKdim}} A & =\underline{\lim }_{n \rightarrow \infty} \frac{\ln \gamma_{A}(n)}{\ln n} .
\end{aligned}
$$

This setting assumes that all elements of $X$ have the same weight equal to 1 . We shall mainly use a somewhat different growth function. Namely, we consider the weight function wt $v, v \in A$, and the growth with respect to it: $\tilde{\gamma}_{A}(n)=\operatorname{dim}_{K}\langle y| y \in$ $A$, wt $y \leq n\rangle, n \in \mathbb{N}$, where the elements of the generating set $X$ have different weights. Standard arguments [14] prove that this growth function yields the same Gelfand-Kirillov dimensions.

Now suppose that char $K=p>0$. Denote $I=\{0,1,2, \ldots\}$ and $\mathbb{N}_{p}=$ $\{0,1, \ldots, p-1\}$. Consider the truncated polynomial algebra

$$
R=K\left[t_{i}, i \in I \mid t_{i}^{p}=0, i \in I\right] .
$$

Let $\mathbb{N}_{p}^{I}=\left\{\alpha: I \rightarrow \mathbb{N}_{p}\right\}$ be the set of functions with finitely many nonzero values. For $\alpha \in \mathbb{N}_{p}^{I}$ denote $|\alpha|=\sum_{i \in I} \alpha_{i}$ and $\boldsymbol{t}^{\alpha}=\prod_{i \in I} t_{i}^{\alpha_{i}} \in R$. The set $\left\{\boldsymbol{t}^{\alpha} \mid \alpha \in \mathbb{N}_{p}^{I}\right\}$ 
is clearly a basis of $R$. Consider the ideal $R^{+}$spanned by all elements $t^{\alpha}, \alpha \in \mathbb{N}_{p}^{I}$, $|\alpha|>0$. Let $\partial_{i}=\frac{\partial}{\partial t_{i}}, i \in I$, denote the partial derivatives of $R$.

We introduce the so-called Lie algebra of special derivations of $R$ [22], [23], [20]:

$$
\boldsymbol{W}(R)=\left\{\sum_{\alpha \in \mathbb{N}_{p}^{I}} \boldsymbol{t}^{\alpha} \sum_{j=1}^{m(\alpha)} \lambda_{\alpha, i_{j}} \frac{\partial}{\partial t_{i_{j}}} \mid \lambda_{\alpha, i_{j}} \in K, i_{j} \in I\right\} .
$$

It is essential that the sum at each $t^{\alpha}, \alpha \in \mathbb{N}_{p}^{I}$, is finite.

Lemma 1.1 ([21]). For arbitrary complex numbers $a_{i} \in \mathbb{C}, i \in \mathbb{N}$, there exist gradations on the algebras $R, \boldsymbol{W}(R)$ such that $\operatorname{wt}\left(t_{i}\right)=-a_{i}, \operatorname{wt}\left(\partial_{i}\right)=a_{i}$.

Denote by $\tau: R \rightarrow R$ the shift endomorphism $\tau\left(t_{i}\right)=t_{i+1}, i \in I$. Extending it by $\tau\left(\partial_{i}\right)=\partial_{i+1}, i \in I$, we get the shift endomorphism $\tau: \boldsymbol{W}(R) \rightarrow \boldsymbol{W}(R)$.

\section{First example}

We define the following two derivations of $R$ :

$$
\begin{aligned}
& v_{1}=\partial_{1}+t_{0}^{p-1}\left(\partial_{2}+t_{1}^{p-1}\left(\partial_{3}+t_{2}^{p-1}\left(\partial_{4}+t_{3}^{p-1}\left(\partial_{5}+t_{4}^{p-1}\left(\partial_{6}+\cdots\right)\right)\right)\right)\right), \\
& v_{2}=\partial_{2}+t_{1}^{p-1}\left(\partial_{3}+t_{2}^{p-1}\left(\partial_{4}+t_{3}^{p-1}\left(\partial_{5}+t_{4}^{p-1}\left(\partial_{6}+\cdots\right)\right)\right)\right) .
\end{aligned}
$$

These operators are special derivations $v_{1}, v_{2} \in \boldsymbol{W}(R)$. Observe that we can write these derivations recursively:

$$
v_{1}=\partial_{1}+t_{0}^{p-1} \tau\left(v_{1}\right), \quad v_{2}=\tau\left(v_{1}\right) .
$$

Let $\boldsymbol{L}=\operatorname{Lie}_{p}\left(v_{1}, v_{2}\right) \subset \boldsymbol{W}(R) \subset$ Der $R$ be the restricted subalgebra generated by $\left\{v_{1}, v_{2}\right\}$. This algebra was introduced in [24]. In the case of characteristic $p=2$, it coincides with the Fibonacci restricted Lie algebra introduced in [19]. Similarly, define

$$
v_{i}=\tau^{i-1}\left(v_{1}\right)=\partial_{i}+t_{i-1}^{p-1}\left(\partial_{i+1}+t_{i}^{p-1}\left(\partial_{i+2}+t_{i+1}^{p-1}\left(\partial_{i+3}+\cdots\right)\right)\right),
$$

$i=1,2, \ldots$ We also can write

$$
v_{i}=\partial_{i}+t_{i-1}^{p-1} v_{i+1}, \quad i=1,2, \ldots
$$

Lemma 2.1. Let $\boldsymbol{L}=\operatorname{Lie}_{p}\left(v_{1}, v_{2}\right) \subset$ Der $R$ be the restricted Lie algebra generated by $\left\{v_{1}, v_{2}\right\}$. Then the following relations holds:

(1) $\left[v_{i}, v_{i+1}\right]=-t_{i}^{p-2} v_{i+2}$ for $i=1,2, \ldots$;

(2) $\left[v_{i}, v_{i+2}\right]=-t_{i-1}^{p-1} t_{i+1}^{p-2} v_{i+3}$ for $i=1,2, \ldots$; 
(3) in general, for all $1 \leq i<j$ we have

$$
\left[v_{i}, v_{j}\right]=-\left(t_{i-1} t_{i} \ldots t_{j-3}\right)^{p-1} t_{j-1}^{p-2} v_{j+1}
$$

(4) for all $n \geq 1, j \geq 0$ we have the action

$$
v_{n}\left(t_{j}\right)= \begin{cases}\left(t_{n-1} t_{n} \cdots t_{j-2}\right)^{p-1}, & n<j \\ 1, & n=j \\ 0, & n>j\end{cases}
$$

(5) for all $k, n \geq 1$,

$$
\left[\partial_{n}, v_{k}\right]= \begin{cases}-\left(t_{k-1} t_{k} \ldots t_{n-1}\right)^{p-1} t_{n}^{p-2} v_{n+2}, & k<n+1, \\ -t_{n}^{p-2} v_{n+2}, & k=n+1, \\ 0, & k>n+1\end{cases}
$$

(6) $v_{i}^{p}=-t_{i-1}^{p-1} v_{i+2}$ for all $i \geq 1$.

Proof. The claims (1)-(5) are proved in [24]. The last claim for $p=2$ is checked in [19], we assume that $p \geq 3$. We have $v_{i}^{p}=\left(\partial_{i}+t_{i-1}^{p-1} v_{i+1}\right)^{p}$. By formula (1) we obtain the sum of commutators of length $p$. We apply the previous claim $\left[\partial_{i}, t_{i-1}^{p-1} v_{i+1}\right]=-t_{i-1}^{p-1} t_{i}^{p-2} v_{i+2}$. In further commutators we cannot use $t_{i-1}^{p-1} v_{i+1}$ anymore because of the total power of $t_{i-1}$. Thus, only one term in (1) is nontrivial, namely $s_{p-1}(x, y)=(\operatorname{ad} x)^{p-1}(y)$. We get

$$
\begin{aligned}
v_{i}^{p} & =\left(\partial_{i}+t_{i-1}^{p-1} v_{i+1}\right)^{p} \\
& =\left(\operatorname{ad} \partial_{i}\right)^{p-1}\left(t_{i-1}^{p-1} v_{i+1}\right) \\
& =\left(\operatorname{ad} \partial_{i}\right)^{p-2}\left(-t_{i-1}^{p-1} t_{i}^{p-2} v_{i+2}\right) \\
& =-t_{i-1}^{p-1} v_{i+2} .
\end{aligned}
$$

Lemma 2.2. Let $H$ be the $K$-linear span of all elements $t_{0}^{\alpha_{0}} t_{1}^{\alpha_{1}} \ldots t_{n-2}^{\alpha_{n-2}} v_{n}$, where $0 \leq \alpha_{i} \leq p-1, \alpha_{n-2} \leq p-2, n \geq 1$. Then $H$ is a restricted subalgebra of Der $R$ and $\boldsymbol{L} \subset H$.

Proof. Let us prove that $H$ is a Lie subalgebra. We apply Lemma 2.1 to check that the product of two monomials of type above is expressed via such monomials again. Let $n<m$. Then

$$
\begin{aligned}
{\left[t_{0}^{\alpha_{0}} \ldots t_{n-2}^{\alpha_{n-2}} v_{n}, t_{0}^{\beta_{0}} \ldots t_{m-2}^{\beta_{m-2}} v_{m}\right] } & \\
= & -t_{0}^{\alpha_{0}} \ldots t_{n-2}^{\alpha_{n-2}}\left(t_{n-1} \ldots t_{m-3}\right)^{p-1} t_{0}^{\beta_{0}} \ldots t_{m-2}^{\beta_{m-2}} t_{m-1}^{p-2} v_{m+1} \\
& \quad+t_{0}^{\alpha_{0}} \ldots t_{n-2}^{\alpha_{n-2}} \sum_{\beta_{j} \neq 0}\left(\prod_{i=0, i \neq j}^{m-2} t_{i}^{\beta_{i}}\right) \beta_{j} t_{j}^{\beta_{j}-1} v_{n}\left(t_{j}\right) v_{m} .
\end{aligned}
$$


The first term is of type $\ldots t_{m-2}^{\beta_{m-2}} t_{m-1}^{p-2} v_{m+1}$, as required.

By claim (4), $v_{m}$ acts on all $t_{i}^{\alpha_{i}}$ s trivially because $m>n>n-2 \geq i$, and no respective terms appear. It remains to consider the second term above. Similarly, $v_{n}\left(t_{j}\right)$ is nonzero only for $n \leq j$, namely

$$
v_{n}\left(t_{j}\right)=\left(t_{n-1} t_{n} \ldots t_{j-2}\right)^{p-1}, \quad n \leq j .
$$

In this case, $n \leq j \leq m-2$ and the new $t_{i}$ 's above have indices such that $n-1<$ $\cdots<j-2 \leq m-4$. We again obtain monomials of the required type. Hence, $H \subset$ Der $R$ is a Lie subalgebra. The last claim of Lemma 2.1 implies that the subalgebra $H \subset$ Der $R$ is restricted.

Let $H_{n}$ denote the $K$-linear span of all elements $t_{0}^{\alpha_{0}} \ldots t_{m-2}^{\alpha_{m-2}} v_{m}$, where $0 \leq \alpha_{i} \leq$ $p-1, \alpha_{m-2} \leq p-2, m \geq n$.

Corollary 2.3. (1) $H_{n} \triangleleft H, n \geq 1 ; H=H_{1} \supset H_{2} \supset \cdots$.

(2) Let $\boldsymbol{L}_{n}=\boldsymbol{L} \cap H_{n}$ for $n \geq 1$. Then the factor algebras $H_{n} / H_{n+2}$ and $\boldsymbol{L}_{n} / \boldsymbol{L}_{n+2}$ are abelian with the trivial p-mapping for all $n \geq 1$.

Proof. The fact that $H_{n}$ are ideals and $H_{n} / H_{n+2}$ are abelian follows from eq. (4) and other arguments of Lemma 2.2. In order to check that the $p$-mapping on $H_{n} / H_{n+2}$ is trivial, we use claim (6) of Lemma 2.1 and eq. (1).

A Lie algebra $L$ is said to be just-infinite if it is infinite-dimensional and any proper factor algebra $L / J$ is finite dimensional.

Lemma 2.4. The algebra $\boldsymbol{L}$ is not just-infinite.

Proof. In the case $p=2$ all elements $\left\{v_{n} \mid n \geq 1\right\}$ belong to $L$; see [19]. In the case of arbitrary characteristic the situation is more complicated, nevertheless, we have $v_{2 n} \in \boldsymbol{L}$ for all $n \geq 1$; see [24]. Now let $J$ be the restricted ideal of $\boldsymbol{L}$ generated by the elements

$$
\left[v_{1}, v_{2 n}\right]=-\left(t_{0} t_{1} \ldots t_{2 n-3}\right)^{p-1} t_{2 n-1}^{p-2} v_{2 n+1}, \quad n \geq 2 .
$$

Observe that they all contain the common factor $t_{0}^{p-1}$, which has no chances to disappear by any further commutation. So, all elements of $J$ have the factor $t_{0}^{p-1}$. Hence, the ideal $J$ is abelian, infinite-dimensional, and has the trivial $p$-mapping. Since the elements $\left\{v_{2 n} \mid n \geq 1\right\}$ are linearly independent modulo $J$, we conclude that $\operatorname{dim} L / J=\infty$.

In our constructions we are motivated by analogies with constructions of selfsimilar groups and algebras [9] [8], [2]. In particular, the following property is analogous to the periodicity of the Grigorchuk and Gupta-Sidki groups [7], [10].

Theorem 2.5 ([19], [24]). Let $\boldsymbol{L}=\operatorname{Lie}_{p}\left(v_{1}, v_{2}\right) \subset$ Der $R$ be the restricted subalgebra generated by $\left\{v_{1}, v_{2}\right\}$. Then $\boldsymbol{L}$ has a nil p-mapping. 


\section{The first example: the gradation}

In this section we introduce a $\mathbb{Z} \oplus \mathbb{Z}$-gradation on our algebras. Suppose that all elements $v_{i}$ are homogeneous, wt $v_{i}=-$ wt $t_{i}=a_{i} \in \mathbb{R}$, where $i=1,2 \ldots$, such that all terms in (3) are homogeneous. To achieve this, we assume that

$$
a_{i}=\mathrm{wt} v_{i}=\mathrm{wt} \partial_{i}=(p-1) \mathrm{wt} t_{i-1}+\mathrm{wt} v_{i+1}=-(p-1) a_{i-1}+a_{i+1} .
$$

Hence, we get the recurrence relation

$$
a_{i+1}=a_{i}+(p-1) a_{i-1}, \quad i \in \mathbb{N} .
$$

This equation has the characteristic polynomial $\phi(t)=t^{2}-t-(p-1)$ with two different roots

$$
\lambda=\frac{1+\sqrt{4 p-3}}{2}, \quad \lambda_{1}=\frac{1-\sqrt{4 p-3}}{2} .
$$

It is well known that all solutions of the recurrence relation (5) are linear combinations of the two sequences $a_{i}=\lambda^{i}, i \in \mathbb{N}$, and $a_{i}=\lambda_{1}^{i}, i \in \mathbb{N}$.

We distinguish two cases.

Irrational: $\lambda, \lambda_{1}$ are irrational, e.g., for primes $p=2,5,11,17,19, \ldots$

Rational: $\lambda, \bar{\lambda}$ are rational (moreover, in this case $\lambda, \lambda_{1}$ are integers), e.g., for primes $p=3,7,13,31,43, \ldots$ Note that in this case $\lambda \in \mathbb{Z}$ and $p=\lambda^{2}-\lambda+1$.

Remark. To the best of our knowledge the question if there are infinitely many such primes is open. A more general question asks whether there are infinitely many primes of the form $a n^{2}+b n+c$, where $a, b, c$ are relatively prime integers, $a$ positive, $a+b$ and $c$ are not both even, and $b^{2}-4 a c$ is not a perfect square; see [11], p. 19.

The existence of two linearly independent weight functions yields a $\mathbb{Z} \oplus \mathbb{Z}$ gradation.

Theorem 3.1. Let $\boldsymbol{L}=\operatorname{Lie}_{p}\left(v_{1}, v_{2}\right) \subset$ Der $R$ be the restricted subalgebra generated by $\left\{v_{1}, v_{2}\right\}$. We introduce weight and superweight functions as follows:

$$
\begin{gathered}
\text { wt } v_{n}=- \text { wt } t_{n}=\lambda^{n}, \quad n=1,2, \ldots, \lambda=\frac{1+\sqrt{4 p-3}}{2}, \\
\text { swt } v_{n}=-\operatorname{swt} t_{n}=\lambda_{1}^{n-2}, \quad n=1,2, \ldots, \lambda_{1}=\frac{1-\sqrt{4 p-3}}{2} .
\end{gathered}
$$

Then:

(1) Both functions are additive on products of homogeneous elements of $\boldsymbol{L}$.

(2) We have the $\mathbb{Z} \oplus \mathbb{Z}$-gradation $\boldsymbol{L}=\bigoplus_{a, b \geq 0} \boldsymbol{L}_{a, b}$, where $\boldsymbol{L}_{a, b}$ is spanned by products with a factors $v_{1}$ and $b$ factors $v_{2}$. 
(3) Let $v \in \boldsymbol{L}_{a, b}$, where $a, b \geq 0$. Then

$$
\text { wt } v=\lambda a+\lambda^{2} b, \quad \text { swt } v=-\frac{\lambda}{p-1} a+b .
$$

Proof. Let us introduce one more function that takes values in $\mathbb{R}^{2}$ :

$$
\mathrm{Wt}\left(v_{i}\right)=-\operatorname{Wt}\left(t_{i}\right)=\left(\operatorname{wt}\left(v_{i}\right), \operatorname{swt}\left(v_{i}\right)\right), \quad i \in \mathbb{N} .
$$

Consider a monomial $v \in \boldsymbol{L}$ that is a product of $a$ elements $v_{1}$ and $b$ elements $v_{2}$. Then both weight functions are well defined on $v$. Moreover, $\mathrm{Wt}(*)$ is additive on products of monomials in $v_{i}$ and $t_{j}$. Therefore, we get

$$
\mathrm{Wt}(v)=a \mathrm{Wt}\left(v_{1}\right)+b \mathrm{Wt}\left(v_{2}\right) .
$$

Consider another pair of integers $\left(a^{\prime}, b^{\prime}\right) \neq(a, b)$ and a monomial $v^{\prime} \in \boldsymbol{L}$ that contains $a^{\prime}, b^{\prime}$ letters $v_{1}, v_{2}$, respectively. By construction,

$$
\begin{aligned}
& \operatorname{Wt}\left(v_{1}\right)=\left(\lambda, \lambda_{1}^{-1}\right)=(\lambda,-\lambda /(p-1)), \\
& \operatorname{Wt}\left(v_{2}\right)=\left(\lambda^{2}, 1\right)=(\lambda+p-1,1) .
\end{aligned}
$$

Since these two vectors are linearly independent over $\mathbb{R}$, we get $\mathrm{Wt}\left(v^{\prime}\right)=a^{\prime} \mathrm{Wt}\left(v_{1}\right)+$ $b^{\prime} \mathrm{Wt}\left(v_{2}\right) \neq \mathrm{Wt}(v)$ and the claimed $\mathbb{Z} \oplus \mathbb{Z}$-gradation $\boldsymbol{L}=\bigoplus_{a, b \geq 0} \boldsymbol{L}_{a, b}$.

Let $v \in \boldsymbol{L}_{a, b}$, where $a, b \geq 0$. Then

$$
\begin{aligned}
\text { wt } v & =a \text { wt } v_{1}+b \text { wt } v_{2}=a \lambda+b \lambda^{2}, \\
\text { swt } v & =a \text { swt } v_{1}+b \text { swt } v_{2}=a \lambda_{1}^{-1}+b=-\frac{\lambda}{p-1} a+b .
\end{aligned}
$$

Let us introduce a new coordinate system on the plane. For a point $A=(x, y) \in$ $\mathbb{R}^{2}$ we define its new coordinates as

$$
\begin{aligned}
& \xi=\operatorname{wt}(x, y)=\lambda x+\lambda^{2} y=\lambda(x+\lambda y), \\
& \eta=\operatorname{swt}(x, y)=-\frac{\lambda}{p-1} x+y=\lambda_{1}^{-1}\left(x+\lambda_{1} y\right) \quad(x, y) \in \mathbb{R}^{2} .
\end{aligned}
$$

We will refer to these coordinates as the weight and the superweight of the point $(x, y)$ respectively. Gradations by superweights yield triangular decompositions.

Corollary 3.2. Consider the restricted Lie algebra $\boldsymbol{L}=\operatorname{Lie}_{p}\left(v_{1}, v_{2}\right)$, the associative algebra $\boldsymbol{A}=\operatorname{Alg}\left(v_{1}, v_{2}\right)$ generated by $v_{1}, v_{2}$, the universal enveloping algebra $U=U(\boldsymbol{L})$, and the universal restricted enveloping algebra $\boldsymbol{u}=u(\boldsymbol{L})$. Then:

(1) All these algebras have decompositions into direct sums of three subalgebras,

$$
\begin{aligned}
\boldsymbol{L} & =\boldsymbol{L}_{+} \oplus \boldsymbol{L}_{0} \oplus \boldsymbol{L}_{-}, \quad \boldsymbol{A}=\boldsymbol{A}_{+} \oplus \boldsymbol{A}_{0} \oplus \boldsymbol{A}_{-}, \\
U & =U_{+} \oplus U_{0} \oplus U_{-}, \quad \boldsymbol{u}=\boldsymbol{u}_{+} \oplus \boldsymbol{u}_{0} \oplus \boldsymbol{u}_{-},
\end{aligned}
$$


where $\boldsymbol{L}_{+}, \boldsymbol{L}_{0}$, and $\boldsymbol{L}_{-}$are spanned by homogeneous elements $v \in \boldsymbol{L}$ such that swt $v>0$, swt $v=0$, and swt $v<0$, respectively. The decompositions of other algebras are defined similarly.

(2) In the irrational case we have $\boldsymbol{L}_{0}=\{0\}, \boldsymbol{A}_{0}=\{0\}, U_{0}=\{0\}, \boldsymbol{u}_{0}=\{0\}$.

Proof. Suppose that $\lambda$ is irrational. Consider $0 \neq v \in \boldsymbol{L}_{a, b}$, where $(a, b) \in \mathbb{Z}^{2}$. Suppose that $\operatorname{swt}(v)=-a \lambda /(p-1)+b=0$. If $b \neq 0$ then $\lambda \in \mathbb{Q}$, a contradiction.

Lemma 3.3. In the irrational case for an arbitrary lattice point $(a, b) \in \mathbb{Z}^{2} \subset \mathbb{R}^{2}$ we have

$$
|\operatorname{wt}(a, b) \cdot \operatorname{swt}(a, b)| \geq \frac{\lambda^{2}}{p-1}, \quad(a, b) \in \mathbb{Z}^{2} .
$$

Proof. Note that the polynomial $\psi(t)=t^{2}+t-(p-1)$ has the discriminant $D=4 p-3$ and no rational roots. For arbitrary integers $a, b \in \mathbb{Z}$ we have $0 \neq$ $|\psi(a / b)|=\left|a^{2}+a b-(p-1) b^{2}\right| b^{-2}$. Hence, $\left|a^{2}+a b-(p-1) b^{2}\right| \geq 1$. By the formulas (6),

$$
\begin{aligned}
|\operatorname{wt}(a, b) \cdot \operatorname{swt}(a, b)| & =\left|\lambda(a+\lambda b) \lambda_{1}^{-1}\left(a+\lambda_{1} b\right)\right| \\
& =\left|\lambda \lambda_{1}^{-1}\right|\left|a^{2}+\left(\lambda+\lambda_{1}\right) a b+\lambda \lambda_{1} b^{2}\right| \\
& =\frac{\lambda^{2}}{p-1}\left|a^{2}+a b-(p-1) b^{2}\right| \geq \frac{\lambda^{2}}{p-1} .
\end{aligned}
$$

Lemma 3.4. Suppose that $p \geq 3$. Let $w=t_{0}^{\alpha_{0}} t_{1}^{\alpha_{1}} \ldots t_{n-2}^{\alpha_{n-2}} v_{n}, n \geq 1$, be a monomial of the subalgebra $H$ above, namely, $0 \leq \alpha_{i} \leq p-1, \alpha_{n-2} \leq p-2$. Then

(1) $\lambda^{n-2} \leq \mathrm{wt}(w) \leq \lambda^{n}$;

(2) $|\operatorname{swt}(w)| \leq C\left|\lambda_{1}\right|^{n-2}$ in case $p \geq 5$;

(3) $|\operatorname{swt}(w)| \leq p n$ in case $p=3$.

Proof. Clearly, wt $w \leq$ wt $v_{n} \leq \lambda^{n}$. In case $n=1$ we have only one monomial $w=v_{1}$ and our estimates are valid. Let $n \geq 2$, we obtain the bounds

$$
\begin{aligned}
\mathrm{wt}(w) & =\operatorname{wt}\left(v_{n}\right)+\sum_{i=0}^{n-2} \alpha_{i} \mathrm{wt} t_{i} \\
& =\lambda^{n}-\sum_{i=0}^{n-2} \alpha_{i} \lambda^{i} \\
& \geq \lambda^{n}-(p-1) \sum_{i=0}^{n-2} \lambda^{i}+\lambda^{n-2} \\
& \geq \lambda^{n}\left(1-\frac{(p-1) \lambda^{-2}}{1-1 / \lambda}+\frac{1}{\lambda^{2}}\right) \\
& =\lambda^{n}\left(\frac{\lambda^{2}-\lambda-(p-1)}{\lambda^{2}-\lambda}+\frac{1}{\lambda^{2}}\right)=\lambda^{n-2} .
\end{aligned}
$$


Claims 2 and 3. In case $p \geq 5$ we have $\left|\lambda_{1}\right|>1$ and get the bound

$$
|\operatorname{swt}(w)|=\left|\operatorname{swt}\left(v_{n}\right)+\sum_{i=0}^{n-2} \alpha_{i} \operatorname{swt} t_{i}\right| \leq\left|\lambda_{1}\right|^{n}+(p-1) \frac{\left|\lambda_{1}\right|^{n-2}}{1-1 /\left|\lambda_{1}\right|}=C\left|\lambda_{1}\right|^{n-2} .
$$

If $p=3$ then $\lambda_{1}=-1$; in this case we have the bound

$$
|\operatorname{swt}(w)|=\left|\operatorname{swt}\left(v_{n}\right)+\sum_{i=0}^{n-2} \alpha_{i} \operatorname{swt} t_{i}\right| \leq 1+(p-1)(n-1) \leq p n .
$$

In [24] it is shown that

$$
\operatorname{GKdim} \boldsymbol{L}=\frac{\ln p}{\ln \lambda}, \quad \mathrm{GKdim} \boldsymbol{A} \leq \frac{2 \ln p}{\ln \lambda},
$$

where $1<\ln p / \ln \lambda<2$. This and the theory of M. Smith [25] imply that the growth of $u(\boldsymbol{L})$ is subexponential and therefore intermediate. Let us determine the growth of $u(\boldsymbol{L})$ more precisely. We will need some definitions. Consider two series of functions $\Phi_{\alpha}^{q}(n), q=2,3$, of natural argument with the parameter $\alpha \in \mathbb{R}^{+}$:

$$
\Phi_{\alpha}^{2}(n)=n^{\alpha}, \quad \Phi_{\alpha}^{3}(n)=\exp \left(n^{\alpha /(\alpha+1)}\right) .
$$

We compare functions $f: \mathbb{N} \rightarrow \mathbb{R}^{+}$by means of the partial order: $f(n) \leq^{\mathrm{a}} g(n)$ if and only if there exists $N>0$ such that $f(n) \leq g(n), n \geq N$. Suppose that $A$ is a finitely generated algebra and $\gamma_{A}(n)$ is its growth function. We define the dimension of level $q, q \in\{2,3\}$, and the lower dimension of level $q$ by

$$
\begin{aligned}
& \operatorname{Dim}^{q} A=\inf \left\{\alpha \in \mathbb{R}^{+} \mid \gamma_{A}(n) \leq^{\mathrm{a}} \Phi_{\alpha}^{q}(n)\right\}, \\
& \underline{\operatorname{Dim}}^{q} A=\sup \left\{\alpha \in \mathbb{R}^{+} \mid \gamma_{A}(n) \geq^{\mathrm{a}} \Phi_{\alpha}^{q}(n)\right\} .
\end{aligned}
$$

The $q$-dimensions for arbitrary level $q \in \mathbb{N}$ were introduced by the first author in order to specify the subexponential growth of universal enveloping algebras [17]. They generalize the Gelfand-Kirillov dimensions. The condition $\operatorname{Dim}^{q} A=$ $\underline{\operatorname{Dim}}^{q} A=\alpha$ means that the growth function $\gamma_{A}(n)$ behaves like $\Phi_{\alpha}^{q}(n)$. The dimensions of level 2 are exactly the upper and lower Gelfand-Kirillov dimensions [6], [14]. The dimensions of level 3 correspond to the superdimensions of [4] up to normalization (see [18]). We describe the growth of $u(\boldsymbol{L})$ in terms of $\operatorname{Dim}^{3} A$.

Lemma 3.5. Let $\theta=\ln p / \ln \lambda$. The growth of the restricted enveloping algebra $u(\boldsymbol{L})$ is intermediate and

$$
1 \leq \operatorname{Dim}^{3} u(\boldsymbol{L}) \leq \theta
$$

Proof. We have $\operatorname{Dim}^{2} \boldsymbol{L}=\mathrm{GKdim} \boldsymbol{L}=\theta$. Now the claim follows from (the proof) of Proposition 1 in [18]. That proposition deals with the growth of the universal enveloping algebra, some minor changes are needed to modify the proof for the restricted enveloping algebra. 


\section{The first example: weight structure}

The weights in the case $p=2$ were studied in [21]. In that case the weights of the algebras $\boldsymbol{L}=\operatorname{Lie}_{p}\left(v_{1}, v_{2}\right)$ and $\boldsymbol{A}=\operatorname{Alg}\left(v_{1}, v_{2}\right)$ lie in the strips $|\eta|<$ const, while the weights of the restricted enveloping algebra $\boldsymbol{u}=\boldsymbol{u}(L)$ are bounded by a parabola-like curve $|\eta| \leq C \xi^{\theta}$, for some constant $0<\theta<1$.

Now we assume that $p \geq 3$. We shall show that the weights of all three algebras $\boldsymbol{L}, \boldsymbol{A}, \boldsymbol{u}$ belong to a region bounded by a parabola-like curve as well.

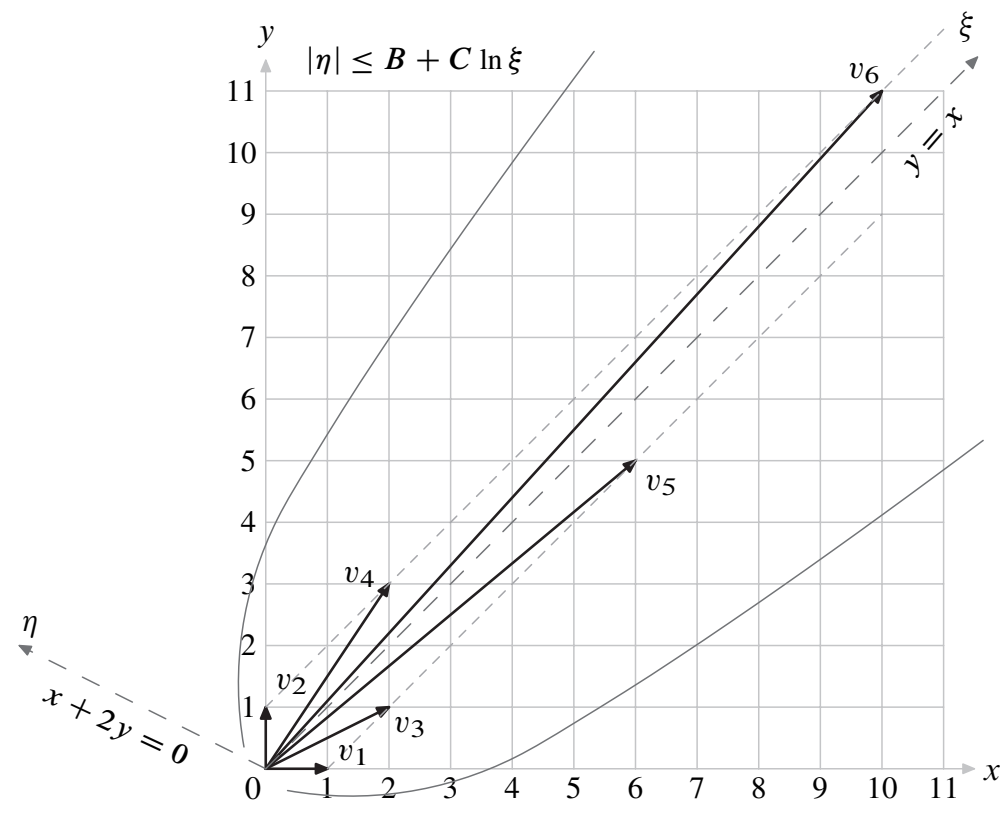

Figure 1. $p=3$, weights of $\boldsymbol{L}$.

Theorem 4.1. Let $p \geq 3$. Consider subalgebras the $H, L$ of Der $R$. Then in terms of the new coordinates $(\xi, \eta)$, homogeneous elements of these algebras belong to the following plane regions.

(1) For $p \geq 5$ we have $|\eta| \leq C \xi^{\theta}$, where $0<\theta=\frac{\ln \left|\lambda_{1}\right|}{\ln \lambda}<1$.

(2) For $p=3$ we have $|\eta| \leq B+C \ln \xi$,

for some positive constants $B, C$.

Proof. Take a basis monomial $w=t_{0}^{\alpha_{0}} t_{1}^{\alpha_{1}} \ldots t_{n-2}^{\alpha_{n-2}} v_{n} \in H$. Consider the new coordinates $(\xi, \eta)$ of $w$. By Lemma 3.4 we have $\xi=\operatorname{wt}(w) \geq \lambda^{n-2}$. Hence, $n \leq 2+\ln \xi / \ln \lambda$. Consider the case $p \geq 5$, we apply the second estimate of 
Lemma 3.4:

$$
|\eta|=|\operatorname{swt}(w)| \leq C\left|\lambda_{1}\right|^{n-2} \leq C\left|\lambda_{1}\right|^{\ln \xi / \ln \lambda}=C \xi^{\ln \left|\lambda_{1}\right| / \ln \lambda} .
$$

In the case $p=3$ we use the third estimate of Lemma 3.4 to get

$$
|\eta|=|\operatorname{swt}(w)| \leq p n \leq p(2+\ln \xi / \ln \lambda) .
$$

Theorem 4.2. Consider the restricted enveloping algebra $\boldsymbol{u}=u(\boldsymbol{L})$. Then there exist constants $C>0$ and $0<\theta<1$ such that homogeneous elements of $\boldsymbol{u}$ belong to the plane region

$$
|\eta| \leq C \xi^{\theta}
$$

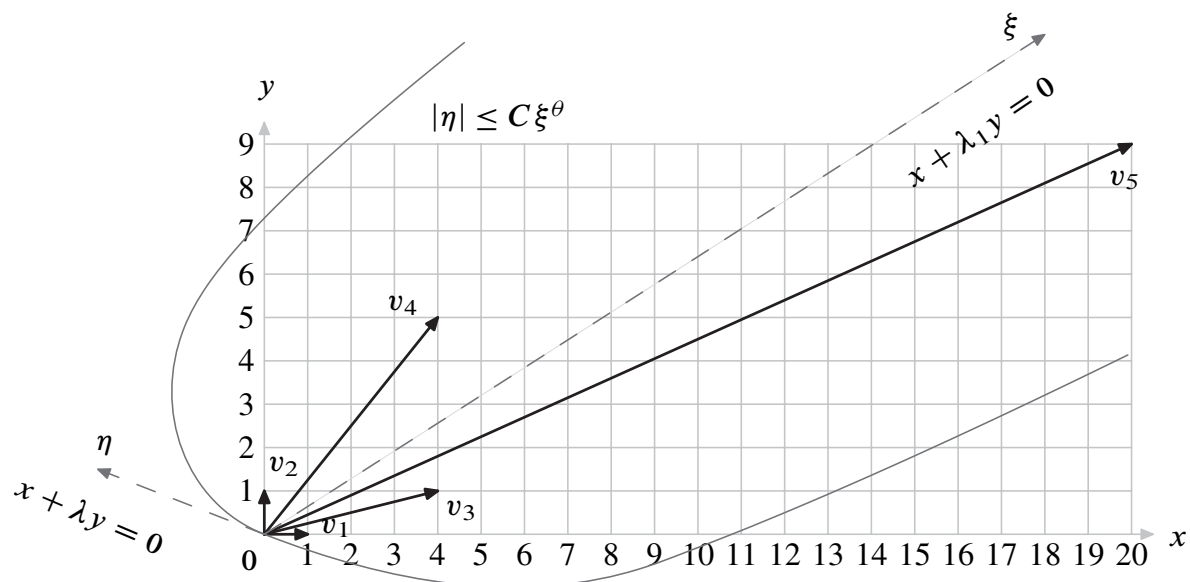

Figure 2. $p=5$, weights of $\boldsymbol{L}$.

Proof. The case $p=2$ was settled in [21]. First, consider the case $p \geq 5$.

We shall consider coordinates of homogeneous elements of the bigger algebra $u(H) \supset \boldsymbol{u}=u(\boldsymbol{L})$. Let $\left\{w_{i} \mid i \in \mathbb{N}\right\}$ be the ordered basis of $H$, which consists of the elements $w=t_{0}^{\alpha_{0}} t_{1}^{\alpha_{1}} \ldots t_{n-2}^{\alpha_{n-2}} v_{n}$, where $0 \leq \alpha_{i} \leq p-1$ and $0 \leq \alpha_{n-2} \leq p-2$. Consider the function $l:\left\{w_{i} \mid i \in \mathbb{N}\right\} \rightarrow \mathbb{N}, l\left(t_{0}^{\alpha_{0}} t_{1}^{\alpha_{1}} \ldots t_{n-2}^{\alpha_{n-2}} v_{n}\right)=n$. Вy Lemma 3.4 we have the estimates

$$
\lambda^{n-2} \leq \operatorname{wt}(w) \leq \lambda^{n}, \quad|\operatorname{swt}(w)| \leq C\left|\lambda_{1}\right|^{n-2}, \quad n \in \mathbb{N} .
$$

Consider a standard basis element of $u(H)$ of type $v=w_{i_{1}} \ldots w_{i_{s}}$, where the $w_{i_{j}}$ enter the product in ordered way and each $w_{j}$ occurs at most $p-1$ times. Let $N=\max \left\{l\left(w_{i_{j}}\right) \mid 1 \leq j \leq s\right\}$. Denote by $\mu_{k}$ the number of $w_{i_{j}}$ such that 
$k=l\left(w_{i_{j}}\right)$, for all $k=1, \ldots, N$. Consider the new coordinates $(\xi, \eta)$, where $\xi=\operatorname{wt}(v)$ and $\eta=\operatorname{swt}(v)$. We apply (7) and obtain the estimates

$$
\frac{1}{\lambda^{2}} \sum_{k=1}^{N} \mu_{k} \lambda^{k} \leq \xi \leq \sum_{k=1}^{N} \mu_{k} \lambda^{k}, \quad|\eta| \leq \frac{C}{\left|\lambda_{1}\right|^{2}} \sum_{k=1}^{N} \mu_{k}\left|\lambda_{1}\right|^{k} .
$$

Let $\alpha=\alpha(v)$ be the number such that $|\eta|=\xi^{\alpha}$. Then

$$
\alpha(v)=\frac{\ln |\eta|}{\ln \xi} \leq \frac{\ln \left(\frac{C}{\left|\lambda_{1}\right|^{2}} \sum_{k=1}^{N} \mu_{k}\left|\lambda_{1}\right|^{k}\right)}{\ln \left(\frac{1}{\lambda^{2}} \sum_{k=1}^{N} \mu_{k} \lambda^{k}\right)} .
$$

The number of different basis elements $w_{i}$ such that $l\left(w_{i}\right)=k$ equals $p^{k-2}(p-1)<$ $p^{k-1}$ for all $k \geq 2$. Each of them can enter $v$ at most $(p-1)$ times. Hence we get

$$
\mu_{k} \leq p^{k-1}(p-1)<p^{k}, \quad k=1, \ldots, N .
$$

Let us evaluate the maximal value of $\alpha(v)$ among all $v$ 's with the fixed value $\xi(v)=\xi_{0}$. From (8) we have $\xi_{0} \leq \sum_{k=1}^{N} \mu_{k} \lambda^{k} \leq \lambda^{2} \xi_{0}$, this estimate yields the range of values for the denominator of (9). To estimate the numerator of (9) we consider the maximum of the linear function

$$
f\left(x_{1}, \ldots, x_{N}\right)=\sum_{k=1}^{N} x_{k}\left|\lambda_{1}\right|^{k}, \quad 0 \leq x_{k} \leq p^{k}, k=1, \ldots, N,
$$

subject to a constraint of the form of a hyperplane $\sum_{k=1}^{N} x_{k} \lambda^{k}=A$, where the constant $A$ is such that $\xi_{0} \leq A \leq \lambda^{2} \xi_{0}$. Note that the denominator of (9) is fixed on each hyperplane. Since $\left|\lambda_{1}\right|<\lambda$, the maximum on each hyperplane is achieved when we assign the biggest possible values for $x_{k}$ with the smallest $k$ 's. By (10), we have the bounds $0 \leq x_{k} \leq p^{k}, k=1, \ldots, N$. Thus, we take the point on the hyperplane $x_{k}=p^{k}, k=1, \ldots, m$, for some $m \leq N$, the appropriate value $x_{k+1} \in\left[0, p^{k+1}\right)$, and $x_{k+2}=\cdots=x_{N}=0$. This point yields the upper bound

$$
\begin{aligned}
\alpha(v) & \leq \frac{\ln \left(\frac{C}{\left|\lambda_{1}\right|^{2}}\left(\sum_{k=1}^{m} p^{k}\left|\lambda_{1}\right|^{k}+x_{k+1}\left|\lambda_{1}\right|^{k+1}\right)\right)}{\ln \left(\frac{1}{\lambda^{2}}\left(\sum_{k=1}^{m}(p \lambda)^{k}+x_{k+1} \lambda^{k+1}\right)\right)} \\
& \leq \frac{\ln \left(C_{1} p^{m}\left|\lambda_{1}\right|^{m}\right)}{\ln \left(C_{2} p^{m} \lambda^{m}\right)}=\frac{\frac{1}{m} \ln C_{1}+\ln \left(p\left|\lambda_{1}\right|\right)}{\frac{1}{m} \ln C_{2}+\ln (p \lambda)} .
\end{aligned}
$$

When $\xi_{0}$ increases, the number $m$ increases as well. Let us choose the number $\theta$ such that $\ln \left(p\left|\lambda_{1}\right|\right) / \ln (p \lambda)<\theta<1$. Then for sufficiently large $\xi$ we have $\alpha(v) \leq \theta$, hence $|\eta| \leq \xi^{\theta}$. By choosing an appropriate constant $C$ we get $|\eta| \leq C \xi^{\theta}$ for all $\xi>0$.

It remains to consider the case $p=3$. For elements of the Lie algebra we have the bound $|\eta| \leq B+C \ln \xi$. Recall that $\lambda_{1}=-1$ in this case. Take $\lambda_{2}=3 / 2<\lambda=2$. 
Let $\left\{w_{i} \mid i \in \mathbb{N}\right\}$ be the ordered basis of $H$ as above. Then we have $\left|\operatorname{swt}\left(w_{i}\right)\right| \leq \lambda_{2}^{n}$, where $n=l\left(w_{i}\right)$, for $i \geq N$, provided that the number $N$ is sufficiently large. We find constant $C_{1}$ such that $\left|\operatorname{swt}\left(w_{i}\right)\right| \leq C_{1} \lambda_{2}^{l\left(w_{i}\right)}$ for all $w_{i}$. Now we can formally apply the arguments above.

Consider the triangular decompositions of Corollary 3.2.

Corollary 4.3. Let $\boldsymbol{L}, \boldsymbol{A}, \boldsymbol{u}$ be as above and consider the decompositions given by the superweight

$$
\boldsymbol{L}=\boldsymbol{L}_{+} \oplus \boldsymbol{L}_{0} \oplus \boldsymbol{L}_{-}, \quad \boldsymbol{A}=\boldsymbol{A}_{+} \oplus \boldsymbol{A}_{0} \oplus \boldsymbol{A}_{-}, \quad \boldsymbol{u}=\boldsymbol{u}_{+} \oplus \boldsymbol{u}_{0} \oplus \boldsymbol{u}_{-} .
$$

(1) Then the upper and lower components $\boldsymbol{L}_{ \pm}, \boldsymbol{A}_{ \pm}, \boldsymbol{u}_{ \pm}$, are locally nilpotent.

(2) In the irrational case, the zero components above are trivial and we obtain decompositions into a direct sum of two locally nilpotent subalgebras.

Proof. Consider, for example, $\boldsymbol{u}_{+}$. The line $\eta=\operatorname{swt}(x, y)=0$ separates the upper and lower components. By (6), this line is given by the equation $x+\lambda_{1} y=$ 0 . Consider homogeneous monomials $u_{1}, \ldots, u_{k} \in \boldsymbol{u}_{+}$above this line and the subalgebra $A=\operatorname{Alg}\left(u_{1}, \ldots, u_{k}\right)$ generated by these elements. Let $N \in \mathbb{N}$ and consider $u=\sum_{j ; n \geq N} \alpha_{j} u_{j_{1}} \ldots u_{j_{n}}, \alpha_{j} \in K$. Then it is geometrically clear (see Figure 3 ) that the respective vectors of all homogeneous components belong to the

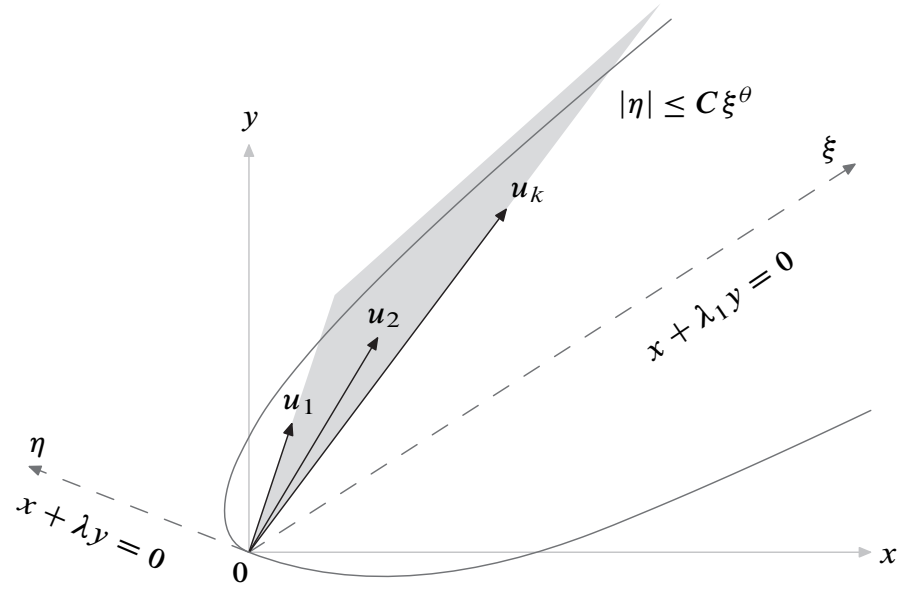

Figure 3. $p \geq 2$, weights of $\boldsymbol{u}$.

shaded angle. All components go out of the region $|\eta|<C \xi^{\theta}$ provided that $N$ is sufficiently large. Hence, $A^{N}=0$.

In irrational case, we obtain some more examples of finitely generated infinitedimensional associative algebras that are direct sums of two locally nilpotent subal- 
gebras. Those examples were constructed in [13], [5]. Our examples, $\boldsymbol{A}$ and $\boldsymbol{u}$, are of polynomial and intermediate growth.

\section{Second example}

Now we turn to the study of the second example suggested in [24]. We keep the same notations $I, R, \boldsymbol{L}, \boldsymbol{A}, H$ as in the first example, but now they denote another objects.

We add some negative indices to the index set $I=\{2-p, 2-p+1, \ldots, 0,1, \ldots\}$ and consider the truncated polynomial ring $R=K\left[t_{i} \mid i \in I\right] /\left(t_{i}^{p} \mid i \in I\right)$. Then we introduce the derivations

$$
v_{m}=\partial_{m}+t_{m-p+1}^{p-1}\left(\partial_{m+1}+t_{m-p+2}^{p-1}\left(\partial_{m+2}+t_{m-p+3}^{p-1}\left(\partial_{m+3}+\ldots\right)\right)\right), \quad m \geq 1 .
$$

As above, $\tau: R \rightarrow R$ is the endomorphism given by $\tau\left(t_{i}\right)=t_{i+1}, i \in I$. Observe that

$$
v_{m}=\partial_{m}+t_{m-p+1}^{p-1} v_{m+1}=\tau^{m-1}\left(v_{1}\right), \quad m \geq 1 .
$$

Now let $\boldsymbol{L}=\operatorname{Lie}_{p}\left(v_{1}, \ldots, v_{p}\right) \subset$ Der $R$ denote the restricted subalgebra generated by $\left\{v_{1}, v_{2}, \ldots, v_{p}\right\}$. In the case of characteristic $p=2$, this example coincides with the Fibonacci restricted Lie algebra [19]. In what follows we assume that $p \geq 3$.

We also can consider a slight modification $\tilde{\boldsymbol{L}}=\operatorname{Lie}_{p}\left(\partial_{1}, \ldots, \partial_{p-1}, v_{p}\right) \subset \operatorname{Der} R$.

Let us make the convention that if the upper index of a product (sum) is less than the lower index, then the product is empty. Similarly, if we list a set as $\{i, i+1, \ldots, j\}$ and $i>j$, then the set is assumed to be empty.

Lemma 5.1. Let $p \geq 3$. The following commutation relations hold:

(1) $\left[v_{m}, v_{m+1}\right]=-\left(\prod_{j=m-p+2}^{m-1} t_{j}^{p-1}\right) t_{m}^{p-2} v_{m+p}$ for $m \geq 1$;

(2) for $n \geq 1, k \geq 2$ we have (both sets in the product below may be empty)

$$
\left[v_{n}, v_{n+k}\right]=-\sum_{j=\max \{0, k-p+1\}}^{k-1}\left(\prod_{\substack{l \in\{1, \ldots, j\} \cup \\ k+1, \ldots, j+p-1\}}} t_{n-p+l}^{p-1}\right) t_{n+j}^{p-2} v_{n+j+p} ;
$$

(3) for all $n, m \geq 1$

$$
\left[\partial_{n}, v_{m}\right]= \begin{cases}-\left(\prod_{j=m-p+1}^{n-1} t_{j}^{p-1}\right) t_{n}^{p-2} v_{n+p}, & m<n+p-1 \\ -t_{n}^{p-2} v_{n+p}, & m=n+p-1 \\ 0, & m>n+p-1\end{cases}
$$

(4) for all $n \geq 1, j \geq 0$ we have the action

$$
v_{m}\left(t_{j}\right)= \begin{cases}\prod_{i=m-p+1}^{j-p} t_{i}^{p-1}, & m<j \\ 1, & m=j \\ 0, & m>j\end{cases}
$$


(5) $v_{n}^{p}=-\left(t_{n-(p-1)} \ldots t_{n-1}\right)^{p-1} v_{n+p}$ for all $n \geq 1$.

Proof. Let us check the first claim:

$$
\begin{gathered}
{\left[v_{m}, v_{m+1}\right]=\left[\partial_{m}+t_{m-p+1}^{p-1} v_{m+1}, v_{m+1}\right]=\left[\partial_{m}, v_{m+1}\right]} \\
=\left[\partial_{m}, \partial_{m+1}+t_{m-p+2}^{p-1}\left(\partial_{m+2}+\cdots\right.\right. \\
\left.\left.\quad \ldots+t_{m-1}^{p-1}\left(\partial_{m+p-1}+t_{m}^{p-1} v_{m+p}\right) \ldots\right)\right] \\
=-t_{m-p+2}^{p-1} \ldots t_{m-1}^{p-1} \cdot t_{m}^{p-2} v_{m+p}
\end{gathered}
$$

To prove the claim (3), observe that the product is nontrivial only for $m \leq n+p-1$. In this case we get

$$
\begin{aligned}
{\left[\partial_{n}, v_{m}\right] } & =\left[\partial_{n}, \partial_{m}+t_{m-p+1}^{p-1}\left(\cdots+t_{n-1}^{p-1}\left(\partial_{n+p-1}+t_{n}^{p-1} v_{n+p}\right) \ldots\right)\right] \\
& =-\left(\prod_{j=m-p+1}^{n-1} t_{j}^{p-1}\right) t_{n}^{p-2} v_{n+p} .
\end{aligned}
$$

Now we prove claim (2). Let $k \geq 2$. We have

$$
\begin{aligned}
{\left[v_{n}, v_{n+k}\right]=} & {\left[\partial_{n}+t_{n-p+1}^{p-1}\left(\partial_{n+1}+\cdots\right.\right.} \\
\left.\left.\cdots+t_{n+k-p-1}^{p-1}\left(\partial_{n+k-1}+t_{n+k-p}^{p-1} v_{n+k}\right) \ldots\right), v_{n+k}\right] & \\
= & {\left[\partial_{n}+t_{n-p+1}^{p-1}\left(\partial_{n+1}+\cdots+t_{n+k-p-1}^{p-1} \partial_{n+k-1}\right), v_{n+k}\right] } \\
= & \sum_{j=0}^{k-1}\left(\prod_{l=1}^{j} t_{n-p+l}^{p-1}\right)\left[\partial_{n+j}, v_{n+k}\right] \\
= & \sum_{j=\max \{0, k-p+1\}}^{k-1}\left(\prod_{l=1}^{j} t_{n-p+l}^{p-1}\right)\left[\partial_{n+j}, \partial_{n+k}\right. \\
= & -\sum_{j=\max \{0, k-p+1\}}^{k-1}\left(\prod_{l=1}^{j} t_{n-p+l}^{p-1}\right)\left(\prod_{q=k+1}^{j+p-1} t_{n-p+q}^{p-1}\right) t_{n+j}^{p-2} v_{n+j+p}^{p-1}
\end{aligned}
$$

Claim 4 is proved as follows:

$$
v_{m}\left(t_{j}\right)=\left(\partial_{m}+t_{m-p+1}^{p-1}\left(\cdots+t_{j-p}^{p-1}\left(\partial_{j}+\ldots\right) \ldots\right)\right)\left(t_{j}\right)=\prod_{i=m-p+1}^{j-p} t_{i}^{p-1}
$$

Consider the last claim.

$$
v_{n}^{p}=\left(\left(\partial_{n}+t_{n+1-p}^{p-1} \partial_{n+1}\right)+t_{n+1-p}^{p-1} t_{n+2-p}^{p-1} v_{n+2}\right)^{p}=(x+y)^{p} .
$$


We have

$$
\begin{aligned}
\operatorname{ad} x(y) & =\operatorname{ad}\left(\partial_{n}+t_{n+1-p}^{p-1} \partial_{n+1}\right)\left(t_{n+1-p}^{p-1} t_{n+2-p}^{p-1} v_{n+2}\right) \\
& =t_{n+1-p}^{p-1} t_{n+2-p}^{p-1} \operatorname{ad}\left(\partial_{n}\right)\left(v_{n+2}\right) \\
& =-t_{n-(p-1)}^{p-1} t_{n-(p-2)}^{p-1}\left(t_{n-(p-3)} \ldots t_{n-1}\right)^{p-1} t_{n}^{p-2} v_{n+p} \\
& =-\left(t_{n-(p-1)} \ldots t_{n-1}\right)^{p-1} t_{n}^{p-2} v_{n+p},
\end{aligned}
$$

where the factor $\left(t_{n-(p-1)} t_{n-(p-2)}\right)^{p-1}$ is present for all $p \geq 3$. We consider all Lie polynomials in $x$ and $y$. We observe that a further multiplication by $y$ is zero due to the total power of the letter $t_{n+1-p}$, which cannot be killed by derivations involved. Thus, only one term in (1) is nontrivial, namely $s_{p-1}(x, y)=(\operatorname{ad} x)^{p-1}(y)$. We get

$$
\begin{aligned}
v_{n}^{p} & =(x+y)^{p} \\
& =(\operatorname{ad} x)^{p-1}(y) \\
& =(\operatorname{ad} x)^{p-2}([x, y]) \\
& =\left(\operatorname{ad}\left(\partial_{n}+t_{n-(p-1)}^{p-1} \partial_{n+1}\right)\right)^{p-2}\left(-\left(t_{n-(p-1)} \ldots t_{n-1}\right)^{p-1} t_{n}^{p-2} v_{n+p}\right) \\
& =\left(\operatorname{ad} \partial_{n}\right)^{p-2}\left(-\left(t_{n-(p-1)} \ldots t_{n-1}\right)^{p-1} t_{n}^{p-2} v_{n+p}\right) \\
& =-\left(t_{n-(p-1)} \ldots t_{n-1}\right)^{p-1} v_{n+p} .
\end{aligned}
$$

Lemma 5.2. Let $H$ be the $K$-linear span of the set

$$
\left\{t_{2-p}^{\alpha_{2-p}} \ldots t_{n-p-1}^{\alpha_{n-p-1}} t_{n-p}^{\alpha_{n-p}} v_{n} \mid 0 \leq \alpha_{i} \leq p-1, \alpha_{n-p} \leq p-2, n \geq 1\right\} .
$$

Then $H$ is a restricted subalgebra of Der $R$ and $\boldsymbol{L} \subset H$.

Proof. We use Lemma 5.1 and proceed as in Lemma 2.2.

\section{Second example: weights}

As above we will define a gradation on the Lie algebra $\boldsymbol{L}$ by presenting it as a direct sum of weight spaces, all weights being complex numbers. We assume that $\operatorname{wt}\left(\partial_{i}\right)=-\operatorname{wt}\left(t_{i}\right)=a_{i} \in \mathbb{C}$ for all $i \in \mathbb{N}$. Let us choose numbers $a_{i}$ so that all terms in the expression of $v_{i}$ are homogeneous. We obtain $a_{i}=$ wt $\partial_{i}=$ wt $\partial_{i+1}+$ $(p-1)$ wt $t_{i-p+1}=a_{i+1}-(p-1) a_{i-p+1}$, which implies the recurrence relation

$$
a_{i}=a_{i-1}+(p-1) a_{i-p}, \quad i \geq p .
$$

Let us study its characteristic polynomial $\phi(x)=x^{p}-x^{p-1}-(p-1)$.

Lemma 6.1. Consider a prime $p \geq 3$ and the polynomial $\phi(x)=x^{p}-x^{p-1}-(p-1)$. Then: 
(1) $\phi(x)$ is irreducible and has distinct roots.

(2) The equation $\phi(x)=0$ has a unique real root $\lambda_{0}$.

(3) Let $\lambda_{1}, \ldots, \lambda_{p-1}$ be the remaining complex roots. Then $1<\left|\lambda_{i}\right|<\lambda_{0}<2$ for all $i=1, \ldots, p-1$.

(4) Let $\left|\lambda_{i}\right|=\left|\lambda_{j}\right|$. Then $i=j$, or $\lambda_{i}=\bar{\lambda}_{j}$.

Proof. Let us prove that $\phi(x)$ is irreducible modulo $p$. Making the substitution $x=1 / y$, we see that it is sufficient to prove that $g(y)=y^{p}-y+1$ is irreducible over $\mathbb{Z}_{p}$. Let $a$ be a root of $g$, then so is $a+1$ and hence $a+i, i=0, \ldots, p-1$, are all roots of $g(y)$. If $m(x)$ is the minimal polynomial of $a$ then $m_{i}(x)=m(x-i)$ is the minimal polynomial of the root $a+i$. Therefore, the minimal polynomials of all roots of $g(y)$ have the same degree $k$, and hence any polynomial that has a common root with $g(y)$ has an irreducible factor of degree $k$. If $g(y)$ is not irreducible then it is a product of several polynomials of degree $k$, which implies that $k$ is a proper factor of $p=\operatorname{deg} g$, a contradiction.

Since the equation is over $\mathbb{Q}$, the roots are distinct.

We consider the derivative $\phi(x)^{\prime}=x^{p-2}(p x-(p-1))$. It has two roots $x_{0}=0$ and $x_{1}=1-1 / p$. Observe that $\phi(0)=\phi(1)=-(p-1)$. Also, $\phi(2)=2^{p-1}-(p-1)>0$. We conclude that $\phi(x)=0$ has a unique real root $\lambda_{0}$, moreover $1<\lambda_{0}<2$.

Now let $\lambda_{1}$ be a root. Recall that $\lambda_{1} \notin \mathbb{R}$. Suppose that $\left|\lambda_{1}\right| \geq \lambda_{0}$. We have two equalities $\lambda_{0}^{p}-\lambda_{0}^{p-1}=p-1$ and $\lambda_{1}^{p}-\lambda_{1}^{p-1}=p-1$, the latter can be depicted as a non-degenerate triangle in $\mathbb{C}$. By the triangle inequality, $p-1>\left|\lambda_{1}\right|^{p}-\left|\lambda_{1}\right|^{p-1}$. Consider the function $f(x)=x^{p}-x^{p-1}, x \in \mathbb{R}$. Using the derivative $f^{\prime}(x)=$ $x^{p-2}(p x-(p-1))$ we see that $f(x)$ is increasing for $x>1-1 / p$. We obtain

$$
p-1=\lambda_{0}^{p}-\lambda_{0}^{p-1}=f\left(\lambda_{0}\right) \leq f\left(\left|\lambda_{1}\right|\right)=\left|\lambda_{1}\right|^{p}-\left|\lambda_{1}\right|^{p-1}<p-1 .
$$

This contradiction proves that $\left|\lambda_{1}\right|<\lambda_{0}$. Suppose that $\left|\lambda_{1}\right| \leq 1$ for a root $\lambda_{1} \notin \mathbb{R}$. Then $p-1=\lambda_{1}^{p}-\lambda_{1}^{p-1}=\left|\lambda_{1}^{p}-\lambda_{1}^{p-1}\right|<2$, a contradiction. Thus $\left|\lambda_{1}\right|>1$ and the third claim is proved.

To prove claim (4), let $\lambda_{1}, \lambda_{2}$ be two different complex roots of our equation such that $\left|\lambda_{1}\right|=\left|\lambda_{2}\right|$. Consider the triangle in $\mathbb{C}$ given by $p-1=\lambda_{1}^{p}-\lambda_{1}^{p-1}$, where $p-1, \lambda_{1}^{p}$ start from the origin. Consider all triangles on the plane with the same side $p-1$, with the other sides of lengths $\left|\lambda_{1}\right|^{p},\left|\lambda_{1}\right|^{p-1}$ and with the longest side starting from the origin. There are only two such triangles. They correspond to $\lambda_{1}$ and $\bar{\lambda}_{1}$. We have two possibilities. a) $\lambda_{1}^{p}=\lambda_{2}^{p}, \lambda_{1}^{p-1}=\lambda_{2}^{p-1}$, and so $\lambda_{1}=\lambda_{2}$. b) $\lambda_{1}^{p}=\bar{\lambda}_{2}^{p}, \lambda_{1}^{p-1}=\bar{\lambda}_{2}^{p-1}$, and we get $\lambda_{1}=\bar{\lambda}_{2}$.

Denote $s=(p-1) / 2$. For simplicity, order the roots so that $\lambda_{i+s}=\bar{\lambda}_{i}$ for $i=1, \ldots s$. We introduce the $p$ weight functions

$$
\mathrm{wt}_{j}\left(\partial_{n}\right)=\lambda_{j}^{n}, \quad n \in \mathbb{N}, j=0, \ldots, p-1 .
$$


By Lemma 1.1, these weight functions define a gradation on the subalgebra $H \subset$ Der $R$ defined above. For a homogeneous element $v \in H$ let

$$
\mathrm{Wt}(v)=\left(\mathrm{wt}_{0} v, \mathrm{wt}_{1} v, \ldots, \mathrm{wt}_{p-1} v\right), \quad v \in H .
$$

Theorem 6.2. Let $\boldsymbol{L}=\operatorname{Lie}_{p}\left(v_{1}, \ldots, v_{p}\right) \subset H \subset \operatorname{Der} R$ be the restricted subalgebras defined above. Then:

(1) The weight functions are additive on products of homogeneous elements of $H$ and $\boldsymbol{L}$.

(2) We have the $\mathbb{Z}^{p}$-gradation

$$
\boldsymbol{L}=\bigoplus_{a_{1}, \ldots, a_{p} \geq 0} \boldsymbol{L}_{a_{1}, \ldots, a_{p}},
$$

where $\boldsymbol{L}_{a_{1}, \ldots, a_{p}}$ is spanned by products with $a_{i}$ factors $v_{i}, i=1, \ldots, p$.

(3) Let $v \in \boldsymbol{L}_{a_{1}, \ldots, a_{p}}$, where $a_{i} \geq 0$. Then

$$
\mathrm{wt}_{j} v=\sum_{k=1}^{p} a_{k} \lambda_{j}^{k}, \quad j=0,1, \ldots, p-1 .
$$

Proof. The additivity follows from Lemma 1.1 and our construction.

Also, by our construction all components of $v_{n}, n \in \mathbb{N}$, have the same weights, namely, $\mathrm{wt}_{j}\left(v_{n}\right)=\mathrm{wt}_{j} \partial_{n}=\lambda_{j}^{n}, j=0,1, \ldots, p-1, n \in \mathbb{N}$. Let $v \in \boldsymbol{L}$ be a monomial that contains $a_{i}$ factors $v_{i}$ for $i=1, \ldots, p$. From additivity of the weight functions we get

$$
\begin{aligned}
\left(\mathrm{wt}_{0} v, \ldots, \mathrm{wt}_{p-1} v\right) & =\mathrm{Wt} v \\
& =\sum_{k=1}^{p} a_{k} \mathrm{Wt}\left(v_{k}\right) \\
& =\sum_{k=1}^{p} a_{k}\left(\lambda_{0}^{k}, \ldots, \lambda_{p-1}^{k}\right) \\
& =\left(\sum_{k=1}^{p} a_{k} \lambda_{0}^{k}, \ldots, \sum_{k=1}^{p} a_{k} \lambda_{p-1}^{k}\right) .
\end{aligned}
$$

The vectors $\operatorname{Wt}\left(v_{k}\right)=\left(\lambda_{0}^{k}, \ldots, \lambda_{p-1}^{k}\right), k=1, \ldots, p$, are linearly independent by Vandermonde's argument. Thus we get the claimed $\mathbb{Z}^{p}$-grading and the third claim as well.

This example also has a nil $p$-mapping.

Theorem 6.3. Let $\boldsymbol{L}=\operatorname{Lie}_{p}\left(v_{1}, v_{2}, \ldots, v_{p}\right) \subset$ Der $R$ be the restricted Lie algebra as above. Then $\boldsymbol{L}$ has a nil p-mapping.

Proof. We refer the reader to the arguments in [24], where it was proved that the $p$-mapping is nil for a class of restricted Lie algebras. 


\section{Second example: triangular decomposition}

Now we want to introduce new coordinates in $\mathbb{R}^{p}$. Let $\bar{x}=\left(x_{1}, \ldots, x_{p}\right) \in \mathbb{R}^{p}$ and set

$$
\begin{aligned}
\xi_{0}(\bar{x}) & =x_{1} \lambda_{0}^{1}+x_{2} \lambda_{0}^{2}+\cdots+x_{p} \lambda_{0}^{p}, \\
\xi_{1}(\bar{x}) & =x_{1} \lambda_{1}^{1}+x_{2} \lambda_{1}^{2}+\cdots+x_{p} \lambda_{1}^{p}, \\
& \vdots \\
\xi_{p-1}(\bar{x}) & =x_{1} \lambda_{p-1}^{1}+x_{2} \lambda_{p-1}^{2}+\cdots+x_{p} \lambda_{p-1}^{p} .
\end{aligned}
$$

Since $\xi_{j}(\bar{x}), \xi_{j+s}(\bar{x})$ are conjugate complex numbers for $j=1, \ldots, s$, we get real coordinates $\left(\eta_{0}, \eta_{1}, \ldots, \eta_{p-1}\right) \in \mathbb{R}^{p}$ as follows (recall that $s=(p-1) / 2$ ). Let $\bar{x}=\left(x_{1}, \ldots, x_{p}\right) \in \mathbb{R}^{p}$ and define

$$
\eta_{k}(\bar{x})= \begin{cases}x_{1} \lambda_{0}^{1}+x_{2} \lambda_{0}^{2}+\cdots+x_{p} \lambda_{0}^{p}, & k=0, \\ \operatorname{Re}\left(x_{1}+x_{2} \lambda_{k}^{1}+\cdots+x_{p} \lambda_{k}^{p-1}\right), & k=1, \ldots, s, \\ \operatorname{Im}\left(x_{1}+x_{2} \lambda_{k}^{1}+\cdots+x_{p} \lambda_{k}^{p-1}\right), & k=s+1, \ldots, p-1 .\end{cases}
$$

We also consider these functions on homogeneous elements $v \in \boldsymbol{L}$. Suppose that $v \in \boldsymbol{L}_{a_{1}, \ldots, a_{p}}$. Then we take $\bar{x}=\left(a_{1}, \ldots, a_{p}\right) \in \mathbb{R}^{p}$ and define

$$
\xi_{j}(v)=\xi_{j}(\bar{x}), \quad \eta_{j}(v)=\eta_{j}(\bar{x}), \quad j=0, \ldots, p-1 .
$$

Lemma 7.1. The introduced weight functions have the following properties:

(1) Let $v \in \boldsymbol{L}_{a_{1}, \ldots, a_{p}}$. Then

$$
\begin{aligned}
\xi_{j}(v)=\mathrm{wt}_{j} v, & j=0, \ldots, p-1, \\
\eta_{0}(v)=\xi_{0}(v)=\mathrm{wt}_{0} v, & \\
\eta_{j}(v)=\operatorname{Re}\left(\mathrm{wt}_{j}(v) / \lambda_{j}\right), & j=1, \ldots, s, \\
\eta_{j}(v)=\operatorname{Im}\left(\mathrm{wt}_{j}(v) / \lambda_{j}\right), & j=s+1, \ldots, p-1 .
\end{aligned}
$$

(2) These functions are additive on products of homogeneous elements of $\boldsymbol{L}$.

(3) Consider a lattice point $\overline{0} \neq \bar{x}=\left(n_{1}, \ldots, n_{p}\right) \in \mathbb{Z}^{p} \subset \mathbb{R}^{p}$. Then $\eta_{j}(\bar{x}) \neq 0$ for all $j=1, \ldots, s$.

(4) Denote $\bar{x}=\left(n_{1}, \ldots, n_{p}\right) \in \mathbb{Z}^{p} \subset \mathbb{R}^{p}$, and let $\eta_{j}(\bar{x})=0$ for some $j \in\{s+1, \ldots, p-1\}$. Then $\bar{x}=\left(n_{1}, 0, \ldots, 0\right)$.

Proof. The first and second claims are obvious.

Let us prove the third claim. Fix $\overline{0} \neq \bar{x}=\left(n_{1}, \ldots, n_{p}\right) \in \mathbb{Z}^{p}$ and $j \in\{1, \ldots, s\}$. Suppose that

$$
\eta_{j}(\bar{x})=\operatorname{Re}\left(n_{1}+n_{2} \lambda_{j}+\cdots+n_{p} \lambda_{j}^{p-1}\right)=0 .
$$


We have the field extension $\mathbb{Q} \subset \mathbb{Q}\left(\lambda_{j}\right)$. Denote $r=n_{1}+n_{2} \lambda_{j}+\cdots+n_{p} \lambda_{j}^{p-1}$. Suppose that $r \neq 0$. From (11) it follows that $r=i q$, where $q \in \mathbb{R}$. Consider $r^{2} \in \mathbb{R} \cap \mathbb{Q}\left(\lambda_{j}\right) \neq \mathbb{Q}\left(\lambda_{j}\right)$. Since $\left|\mathbb{Q}\left(\lambda_{j}\right): \mathbb{Q}\right|=p$ is a prime, we obtain $r^{2} \in \mathbb{Q}$. Then $|\mathbb{Q}(r): \mathbb{Q}|=2$ divides $p$, a contradiction. Therefore, $r=n_{1}+n_{2} \lambda_{j}+$ $\cdots+n_{p} \lambda_{j}^{p-1}=0$, which is a contradiction to the fact that $\lambda_{j}$ satisfies an irreducible polynomial of degree $p$.

We now turn to claim (4). Fix $\bar{x}=\left(n_{1}, \ldots, n_{p}\right) \in \mathbb{Z}^{p}$ and $j \in\{s+1, \ldots, p-1\}$. Suppose that

$$
\eta_{j}(\bar{x})=\operatorname{Im}\left(n_{1}+n_{2} \lambda_{j}+\cdots+n_{p} \lambda_{j}^{p-1}\right)=0 .
$$

Denote $r=n_{1}+n_{2} \lambda_{j}+\cdots+n_{p} \lambda_{j}^{p-1}$. Then $r \in \mathbb{R} \cap \mathbb{Q}\left(\lambda_{j}\right) \neq \mathbb{Q}\left(\lambda_{j}\right)$. Since $\left|\mathbb{Q}\left(\lambda_{j}\right): \mathbb{Q}\right|=p$ is a prime, we get $r \in \mathbb{Q}$. We obtain $\left(n_{1}-r\right)+n_{2} \lambda_{j}+\cdots+$ $n_{p} \lambda_{p}^{p-1}=0$, which is possible only in the case $n_{1}=r, n_{2}=\cdots=n_{p}=0$.

Now we get triangular decompositions where the zero component is always trivial.

Corollary 7.2. Let $\boldsymbol{L}=\operatorname{Lie}_{p}\left(v_{1}, \ldots, v_{p}\right)$, let $\boldsymbol{A}=\operatorname{Alg}\left(v_{1}, \ldots, v_{p}\right)$ be the restricted Lie algebra and associative algebra generated by $\left\{v_{1}, \ldots, v_{p}\right\}$. Let $U=U(\boldsymbol{L})$, $\boldsymbol{u}=u(\boldsymbol{L})$ be the universal enveloping algebra and the restricted enveloping algebra. Then all these algebras have decompositions into direct sums of two subalgebras as follows:

$$
\boldsymbol{L}=\boldsymbol{L}_{+} \oplus \boldsymbol{L}_{-}, \quad \boldsymbol{A}=\boldsymbol{A}_{+} \oplus \boldsymbol{A}_{-}, \quad U=U_{+} \oplus U_{-}, \quad \boldsymbol{u}=\boldsymbol{u}_{+} \oplus \boldsymbol{u}_{-} .
$$

Proof. Fix $j \in\{1, \ldots, s\}$ and set, for example,

$$
\boldsymbol{L}_{+}=\left\langle v \in \boldsymbol{L} \mid \eta_{j}(v)>0\right\rangle, \quad \boldsymbol{L}_{-}=\left\langle v \in \boldsymbol{L} \mid \eta_{j}(v)<0\right\rangle .
$$

Observe that the weight functions $\eta_{j}, j \in\{s+1, \ldots, p-1\}$, also yield triangular decompositions, but in this case the components $\boldsymbol{L}_{0}$ and $\boldsymbol{A}_{0}$ are nontrivial and finite dimensional. Indeed, consider $\boldsymbol{L}_{0}=\left\langle v \in \boldsymbol{L} \mid \eta_{j}(v)=0\right\rangle$. By claim (4) of Lemma 7.1, $\boldsymbol{L}_{0}$ is spanned by products of the element $v_{1}$ only. Since $v_{1}^{p^{2}}=0$, we conclude that $\boldsymbol{L}_{0}=\left\langle v_{1}, v_{1}^{p}\right\rangle$, similarly, $\boldsymbol{A}_{0}=\left\langle v_{1}^{j} \mid 1 \leq j<p^{2}\right\rangle$ is of dimension at most $p^{2}$.

Lemma 7.3. Let $v=t_{2-p}^{\alpha_{2-p}} \ldots t_{n-p}^{\alpha_{n-p}} v_{n} \in H, n \in \mathbb{N}$, be as in Lemma 5.2. Then

(1) $\lambda_{0}^{n-p} \leq \mathrm{wt}_{0} v \leq \lambda_{0}^{n}$;

(2) $\left|\mathrm{wt}_{j} v\right| \leq C\left|\lambda_{j}\right|^{n}$ for all $j=1, \ldots, p-1$, where $C$ is some constant;

(3) $\left|\eta_{j}(v)\right| \leq C\left|\lambda_{j}\right|^{n}$ for all $j=1, \ldots, p-1$. 
Proof. The upper bound $\mathrm{wt}_{0} v \leq \lambda_{0}^{n}$ is obvious. We check the lower bound. Recall that $\alpha_{n-p} \leq p-2$. Then

$$
\begin{aligned}
\mathrm{wt}_{0}(v) & =\lambda_{0}^{n}-\sum_{i=2-p}^{n-p} \alpha_{i} \lambda_{0}^{i} \\
& \geq \lambda_{0}^{n}-(p-1) \sum_{i=2-p}^{n-p} \lambda_{0}^{i}+\lambda_{0}^{n-p} \\
& \geq \lambda_{0}^{n}-(p-1) \frac{\lambda_{0}^{n-p}}{1-1 / \lambda_{0}}+\lambda_{0}^{n-p} \\
& =\frac{\lambda_{0}^{n-p}\left(\lambda_{0}^{p}-\lambda_{0}^{p-1}-(p-1)\right)}{1-1 / \lambda_{0}}+\lambda_{0}^{n-p}=\lambda_{0}^{n-p} .
\end{aligned}
$$

Similarly,

$$
\begin{aligned}
\left|\mathrm{wt}_{j}(v)\right| & =\left|\lambda_{j}^{n}-\sum_{i=2-p}^{n-p} \alpha_{i} \lambda_{j}^{i}\right| \\
& \leq\left|\lambda_{j}\right|^{n}+(p-1) \sum_{i=2-p}^{n-p}\left|\lambda_{j}\right|^{i} \\
& \leq\left|\lambda_{j}\right|^{n}+(p-1) \frac{\left|\lambda_{j}\right|^{n-p}}{1-1 /\left|\lambda_{j}\right|} \leq C\left|\lambda_{j}^{n}\right| .
\end{aligned}
$$

The third claim follows by the previous lemma.

Now we are going to show that the weights of all three algebras $\boldsymbol{L}, \boldsymbol{A}, \boldsymbol{u}$ again belong to a paraboloid-like region of $\mathbb{R}^{p}$ stretched along the axis $\eta_{0}$.

Theorem 7.4. Let $p \geq 3$ and $\boldsymbol{L}=\operatorname{Lie}_{p}\left(v_{1}, \ldots, v_{p}\right), H$ be the subalgebras of Der $R$ as above. Then the new coordinates $\left(\eta_{0}, \eta_{1}, \ldots, \eta_{p-1}\right)$ of homogeneous elements of these algebras belong to the following region of $\mathbb{R}^{p}$ :

$$
\left|\eta_{j}\right| \leq C \eta_{0}^{\theta_{j}}, \quad \theta_{j}=\frac{\ln \left|\lambda_{j}\right|}{\ln \lambda_{0}}<1, \quad j=1, \ldots, p-1,
$$

where $C$ is a positive constant.

Proof. Take a basic monomial $w=t_{2-p}^{\alpha_{2-p}} \ldots t_{n-2}^{\alpha_{n-2}} v_{n} \in H$ and consider its new coordinates $\left(\eta_{0}, \eta_{1}, \ldots, \eta_{p-1}\right)$. By Lemma 7.3 , we have $\eta_{0}=\operatorname{wt}_{0}(w) \geq \lambda_{0}^{n-p}$. Hence, $n \leq p+\ln \eta_{0} / \ln \lambda_{0}$. We apply the third estimate of Lemma 7.3

$$
\left|\eta_{j}\right| \leq C\left|\lambda_{j}\right|^{n} \leq C\left|\lambda_{j}\right|^{p+\ln \eta_{0} / \ln \lambda_{0}}=\tilde{C} \eta_{0}^{\ln \left|\lambda_{j}\right| / \ln \lambda_{0}}, \quad j=1, \ldots, p-1
$$


Theorem 7.5. Let $p \geq 3$. Consider $\boldsymbol{A}=\operatorname{Alg}\left(v_{1}, \ldots, v_{p}\right)$ and $\boldsymbol{u}=u(\boldsymbol{L})$. Then the new coordinates of homogeneous elements of these algebras also belong to the following region of $\mathbb{R}^{p}$ :

$$
\left|\eta_{j}\right| \leq C \eta_{0}^{\theta}, \quad j=1, \ldots, p-1
$$

for some constants $C>0$ and $0<\theta<1$.

Proof. Let $v=t_{2-p}^{\alpha_{2-p}} \ldots t_{n-p}^{\alpha_{n-p}} v_{n} \in H, n \in \mathbb{N}$. By Lemma 7.3 we have bounds similar to (7),

$$
\lambda_{0}^{n-p} \leq \mathrm{wt}_{0} v \leq \lambda_{0}^{n}, \quad\left|\eta_{j}(v)\right| \leq C\left|\lambda_{j}\right|^{n}, \quad\left|\lambda_{j}\right|<\lambda_{0}, j=1, \ldots, p-1 .
$$

It remains to repeat the arguments of Theorem 4.2.

Corollary 7.6. Consider the triangular decompositions of Corollary 7.2,

$$
\boldsymbol{L}=\boldsymbol{L}_{+} \oplus \boldsymbol{L}_{-}, \quad \boldsymbol{A}=\boldsymbol{A}_{+} \oplus \boldsymbol{A}_{-}, \quad \boldsymbol{u}=\boldsymbol{u}_{+} \oplus \boldsymbol{u}_{-} .
$$

Then all the components $\boldsymbol{L}_{ \pm}, \boldsymbol{A}_{ \pm}$, and $\boldsymbol{u}_{ \pm}$are locally nilpotent subalgebras.

Proof. The arguments of Corollary 4.3 apply.

\section{Second example: growth}

In this section we study the growth of the algebras that appear in the second example. In particular we check that $\boldsymbol{L}$ is infinite-dimensional.

Theorem 8.1. Let $\boldsymbol{L}=\operatorname{Lie}_{p}\left(v_{1}, \ldots, v_{p}\right)$, and let $\lambda_{0}$ be the root of the characteristic polynomial above. Then $\mathrm{GKdim} L \leq \ln p / \ln \lambda_{0}$.

Proof. We use the embedding of Lemma 5.2. Fix a number $m$. Consider a homogeneous element $g \in \boldsymbol{L} \subset H$ such that $\mathrm{wt}_{0}(g) \leq m$. Then it is a sum of monomials $v=t_{2-p}^{\alpha_{2-p}} \ldots t_{n-p}^{\alpha_{n-p}} v_{n}$, where $0 \leq \alpha_{i} \leq p-1$ and $\alpha_{n-p} \leq p-2$. By Lemma 7.3, $m \geq \mathrm{wt}_{0}(g) \geq \lambda_{0}^{n-p}$. Hence, $n \leq n_{0}=p+\left[\ln m / \ln \lambda_{0}\right]$.

We estimate the number of monomials $v$ of weight not exceeding $m$ and obtain the bound

$$
\tilde{\gamma}_{\boldsymbol{L}}(m) \leq \sum_{n=1}^{n_{0}} p^{n-1} \leq \frac{p^{n_{0}-1}}{1-1 / p} \leq \frac{p^{p-1+\ln m / \ln \lambda_{0}}}{1-1 / p} \approx C_{0} m^{\ln p / \ln \lambda_{0}}
$$

Corollary 8.2. Let $\boldsymbol{L}=\operatorname{Lie}_{p}\left(v_{1}, \ldots, v_{p}\right), \lambda_{0}$ as above and $\theta=\ln p / \ln \lambda_{0}$. Then the growth of the restricted enveloping algebra $u(\boldsymbol{L})$ is intermediate and

$$
1 \leq \operatorname{Dim}^{3} u(\boldsymbol{L}) \leq \theta
$$


Proof. The result follows by Proposition 1 of [18].

Theorem 8.3. Let $\boldsymbol{A}=\operatorname{Alg}\left(v_{1}, \ldots, v_{p}\right)$. Then GKdim $\boldsymbol{A} \leq 2 \ln p / \ln \lambda_{0}$, where $\lambda_{0}$ is as above.

Proof. We embed our algebra into a bigger associative subalgebra $\boldsymbol{A} \subset \operatorname{Alg}(H) \subset$ $\operatorname{End}(R)$, where $H$ was defined in Lemma 5.2. We claim that elements of $\operatorname{Alg}(H)$ can be expressed as linear combinations of the monomials

$$
w=t_{2-p}^{\alpha_{2-p}} \ldots t_{n-p}^{\alpha_{n-p}} v_{1}^{\beta_{1}} \ldots v_{n}^{\beta_{n}}, \quad 0 \leq \alpha_{i}, \beta_{i} \leq p-1, \beta_{n} \geq 1, n \in \mathbb{N},
$$

where in case $\beta_{n}=1$ we additionally assume that $\alpha_{n-p} \leq p-2$.

Indeed, let us consider a product $w=u_{1} \ldots u_{s}$ of basis monomials $u_{i}=$ $t_{2-p}^{\gamma_{2}-p} \ldots t_{m_{i}-p}^{\gamma_{m_{i}-p}} v_{m_{i}} \in H$, where $\gamma_{m_{i}-p} \leq p-2, i=1, \ldots, s$. Consider the largest index $M(w)=\max \left\{m_{i} \mid i=1, \ldots, s\right\}$. Then the highest $t_{i}$ is $t_{M-p}$. Our product satisfies the following property VTmax: if the highest $v_{M}$ is unique in the product, then the highest variable $t_{M-p}$ has the total occurrence at most $p-2$. We straighten the product to the form (12). Let us check that VTmax is kept under the process. We perform the following transformations.

Case 1. $v_{n} v_{m}=v_{m} v_{n}+\left[v_{n}, v_{m}\right]$ if $n>m$. Consider the terms of the product $\left[v_{n}, v_{m}\right]$, see Lemma 5.1, claims (1), (2). If we get a new highest $v_{M^{\prime}}$, we obtain the highest $t_{M^{\prime}-p}$ in degree $p-2$ as well, the property VTmax is kept. If we get one more term $v_{M}$, then there is nothing to check. If we obtain $v_{j}$ such that $j<M$, then we get at most $t_{j-p}$, and the total degree of the highest $t_{M-p}$ is not changed, as required.

Case 2. $v_{n}^{p}$ is expressed as in claim (5) of Lemma 5.1. We can only get a new highest $v_{M^{\prime}}$ with no occurrence of $t_{M^{\prime}-p}$ at all.

Case 3. The remaining operation is $v_{n} t_{i}=t_{i} v_{n}+v_{n}\left(t_{i}\right)$. Observe the second term. This operation cannot kill the highest $v_{M}$ since $i \leq M-p<M$. Also, $t_{i}$ is replaced by a product of smaller $t_{j}$ s only. Thus, VTmax is kept.

Finally, we arrive at a monomial of type (12), the property VTmax means that in the case $\beta_{n}=1$ we have $\alpha_{n-p} \leq p-2$. Thus, $\operatorname{Alg}(H)$ is spanned by the claimed monomials.

Let us estimate the weight of a monomial (12). In case $\beta_{n}=1$, we use the fact that $\alpha_{n-p} \leq p-2$ and obtain, as in Lemma 7.3, the estimate

$$
\begin{aligned}
\mathrm{wt}_{0}(w) & \geq \lambda_{0}^{n}-\sum_{i=2-p}^{n-p} \alpha_{i} \lambda_{0}^{i} \geq \lambda_{0}^{n}-(p-1) \sum_{i=2-p}^{n-p} \lambda_{0}^{i}+\lambda_{0}^{n-p} \\
& \geq \lambda_{0}^{n}-(p-1) \frac{\lambda_{0}^{n-p}}{1-1 / \lambda_{0}}+\lambda_{0}^{n-p} \\
& =\frac{\lambda_{0}^{n-p}\left(\lambda_{0}^{p}-\lambda_{0}^{p-1}-(p-1)\right)}{1-1 / \lambda_{0}}+\lambda_{0}^{n-p}=\lambda_{0}^{n-p} .
\end{aligned}
$$


In the case $\beta_{n}>1$ we have

$$
\mathrm{wt}_{0}(w) \geq 2 \lambda_{0}^{n}-(p-1) \sum_{i=2-p}^{n-p} \lambda_{0}^{i} \geq \lambda_{0}^{n}-(p-1) \sum_{i=2-p}^{n-p} \lambda_{0}^{i}+\lambda_{0}^{n-p} \geq \lambda_{0}^{n-p} .
$$

Fix a number $m$. Consider all monomials $w$ of type (12) such that $\mathrm{wt}_{0}(w) \leq m$. Both cases above yield the estimate $m \geq \mathrm{wt}_{0}(w) \geq \lambda_{0}^{n-p}$. Then $n \leq n_{0}=p+$ $\left[\ln m / \ln \lambda_{0}\right]$.

Now we can estimate the number of monomials $w$ of weight not exceeding $m$ and obtain the bound

$$
\tilde{\gamma}_{\boldsymbol{A}}(m) \leq \sum_{n=1}^{n_{0}} p^{2 n-1} \leq \frac{p^{2 n_{0}-1}}{1-1 / p^{2}} \leq \frac{p^{2 \ln m / \ln \lambda_{0}+2 p-1}}{1-1 / p^{2}} \approx C_{0} m^{2 \ln p / \ln \lambda_{0}} .
$$

Let us prove the following commutation relation.

Lemma 8.4. For all $n \geq 1$ we have

$$
\begin{aligned}
&\left(\operatorname{ad} v_{n}\right)^{p-1}\left(v_{n+p-1}\right) \\
&=-v_{n+p} \\
&-t_{n}\left(t_{n-p+1}\right)^{p-1} \cdot t_{n+1}^{p-2} v_{n+p+1} \\
&-t_{n}\left(t_{n-p+1} t_{n-p+2} \cdot t_{n+1}\right)^{p-1} \cdot t_{n+2}^{p-2} v_{n+p+2} \\
& \vdots \\
&-t_{n}\left(t_{n-p+1} \ldots t_{n-2} \cdot t_{n+1} \ldots t_{n+p-3}\right)^{p-1} \cdot t_{n+p-2}^{p-2} v_{n+2 p-2} \\
&-2 t_{n}\left(t_{n-p+1} \ldots t_{n-1} \cdot t_{n+1} \ldots t_{n+p-2}\right)^{p-1} \cdot t_{n+p-1}^{p-2} v_{n+2 p-1} .
\end{aligned}
$$

Proof. In claim (2) of Lemma 5.1 we take $k=p-1$

$$
\begin{aligned}
{\left[v_{n}, v_{n+p-1}\right]=} & -\sum_{j=0}^{p-2}\left(\prod_{\substack{\{\in\{1, \ldots, j\} \cup 1 \\
\{p, \ldots, p+j-1\}}} t_{n-p+l}^{p-1}\right) t_{n+j}^{p-2} v_{n+j+p} \\
= & -t_{n}^{p-2} v_{n+p} \\
& -\left(t_{n-p+1} \cdot t_{n}\right)^{p-1} \cdot t_{n+1}^{p-2} v_{n+p+1} \\
& -\left(t_{n-p+1} t_{n-p+2} \cdot t_{n} t_{n+1}\right)^{p-1} \cdot t_{n+2}^{p-2} v_{n+p+2} \\
& \vdots \\
& -\left(t_{n-p+1} t_{n-p+2} \ldots t_{n-2} \cdot t_{n} t_{n+1} \ldots t_{n+p-3}\right)^{p-1} \cdot t_{n+p-2}^{p-2} v_{n+2 p-2} .
\end{aligned}
$$

Let us further commute this expression with $v_{n}$. Recall that $v_{n}$ acts trivially on $t_{n-p+1}, \ldots t_{n-2}$. By Lemma 5.1 all elements $v_{n}\left(t_{j}\right)$, where $j \geq n+1$, contain the factor $t_{n-p+1}^{p-1}$ and we get zero due to the other factor $t_{n-p+1}^{p-1}$. The same argument 
applies to $\left[v_{n}, v_{j}\right]$, where $j \geq n+p+1$. Thus, we get a nontrivial action only in the cases $v_{n}\left(t_{n}\right)=1$ and $\left[v_{n}, v_{n+p}\right]$. Therefore when commuted with $v_{n}$ all terms in the sum above except the first one change only the power of $t_{n}$. Considering the first term we take into account that

$$
\begin{aligned}
{\left[v_{n}, v_{n+p}\right]=} & -\left(t_{n-p+1}\right)^{p-1} \cdot t_{n+1}^{p-2} v_{n+p+1} \\
& -\left(t_{n-p+1} t_{n-p+2} \cdot t_{n+1}\right)^{p-1} \cdot t_{n+2}^{p-2} v_{n+p+2} \\
& -\left(t_{n-p+1} t_{n-p+2} t_{n-p+3} \cdot t_{n+1} t_{n+2}\right)^{p-1} \cdot t_{n+3}^{p-2} v_{n+p+3} \\
& \vdots \\
& -\left(t_{n-p+1} t_{n-p+2} \ldots t_{n-1} \cdot t_{n+1} t_{n+2} \ldots t_{n+p-2}\right)^{p-1} \cdot t_{n+p-1}^{p-2} v_{n+2 p-1}
\end{aligned}
$$

Each time when commuting the first term of (14) with $v_{n}$, these summands add to the existing ones. As a result, there exist some scalars $B_{s, j}$ for $s=1, \ldots, p-1$, $j=1, \ldots, p-1$ such that

$$
\begin{aligned}
\left(\operatorname{ad} v_{n}\right. & )^{s}\left(v_{n+p-1}\right) \\
= & (-1)(-2) \ldots(-s) t_{n}^{p-s-1} v_{n+p} \\
& +B_{s, 1} t_{n}^{p-s}\left(t_{n-p+1}\right)^{p-1} t_{n+1}^{p-2} v_{n+p+1} \\
& +B_{s, 2} t_{n}^{p-s}\left(t_{n-p+1} t_{n-p+2} \cdot t_{n+1}\right)^{p-1} \cdot t_{n+2}^{p-2} v_{n+p+2} \\
& \vdots \\
& +B_{s, p-2} t_{n}^{p-s}\left(t_{n-p+1} t_{n-p+2} \ldots t_{n-2} \cdot t_{n+1} \ldots t_{n+p-3}\right)^{p-1} \cdot t_{n+p-2}^{p-2} v_{n+2 p-2} \\
& +B_{s, p-1} t_{n}^{p-s}\left(t_{n-p+1} t_{n-p+2} \ldots t_{n-1} \cdot t_{n+1} \ldots t_{n+p-2}\right)^{p-1} \cdot t_{n+p-1}^{p-2} v_{n+2 p-1}
\end{aligned}
$$

We have the recurrence relations $B_{s+1, j}=-s B_{s, j}-(-1)^{s} s !, s \geq 1$ for all $j=$ $1, \ldots, p-1$ and the original conditions $B_{1,1}=B_{1,2}=\cdots=B_{1, p-2}=-1$ and $B_{1, p-1}=0$. We check that for all $j=1, \ldots, p-2$ we get $B_{s, j}=(-1)^{s} s !, s \geq 1$; in particular, $B_{p-1, j}=-1$. For $j=p-1$ we have $B_{s, p-1}=(-1)^{s}(s-1)(s-1)$ !, $s \geq 1$, in particular $B_{p-1, p-1}=-2$.

Let us introduce the following convenient notations. Let $v=\sum_{i \geq m} a_{i} v_{i} \in H$, where $a_{i} \in R$. Then we write $v=O\left(v_{m}\right)$. Also suppose that $r_{1}, \ldots, r_{s} \in R$. Then denote by $O\left(\left(r_{1}, \ldots, r_{s}\right) v_{m}\right)$ an element $h \in H$ of the form

$$
h=\sum_{i=1}^{s} r_{i} g_{i}, \quad g_{i}=O\left(v_{m}\right) .
$$

Lemma 8.5. For all $m \geq 1$ we have $\left[H, O\left(v_{m}\right)\right]=O\left(v_{m}\right)$.

Proof. Follows from the commutation relations of Lemma 5.1. 
Lemma 8.6. Let $\boldsymbol{L}=\operatorname{Lie}_{p}\left(v_{1}, \ldots, v_{p}\right)$. Then there exist homogeneous elements of the form

$$
\tilde{v}_{n}=v_{n}+O\left(\left(t_{2-p}^{p-1}, \ldots, t_{n-2 p+1}^{p-1}\right) v_{n+1}\right) \in \boldsymbol{L}, \quad n=1,2, \ldots
$$

Proof. We begin with $\tilde{v}_{1}=v_{1}, \ldots, \tilde{v}_{p}=v_{p}$. Assume that all elements $\tilde{v}_{i}$, with $i \leq n+p-1$, are defined. By assumption we have elements

$$
\begin{aligned}
\tilde{v}_{n} & =v_{n}+O\left(\left(t_{2-p}^{p-1}, \ldots, t_{n-2 p+1}^{p-1}\right) v_{n+1}\right) \in \boldsymbol{L}, \\
\tilde{v}_{n+p-1} & =v_{n+p-1}+O\left(\left(t_{2-p}^{p-1}, \ldots, t_{n-p}^{p-1}\right) v_{n+p}\right) \in \boldsymbol{L} .
\end{aligned}
$$

Consider $\left[\tilde{v}_{n}, \tilde{v}_{n+p-1}\right]$. We use (13) and the commutation relations of Lemma 5.1 to get

$$
\begin{aligned}
& {\left[v_{n}, v_{n+p-1}\right]=-t_{n}^{p-2} v_{n+p}+t_{n-p+1}^{p-1} O\left(v_{n+p+1}\right),} \\
& {\left[v_{n}, O\left(\left(t_{2-p}^{p-1}, \ldots, t_{n-p}^{p-1}\right) v_{n+p}\right)\right]=O\left(\left(t_{2-p}^{p-1}, \ldots, t_{n-p}^{p-1}\right) v_{n+p}\right),} \\
& {\left[v_{n+p-1}, O\left(\left(t_{2-p}^{p-1}, \ldots, t_{n-2 p+1}^{p-1}\right) v_{n+1}\right)\right]=O\left(\left(t_{2-p}^{p-1}, \ldots, t_{n-2 p+1}^{p-1}\right) v_{n+p+1}\right),} \\
& {\left[O\left(\left(t_{2-p}^{p-1}, \ldots, t_{n-2 p+1}^{p-1}\right) v_{n+1}\right), O\left(\left(t_{2-p}^{p-1}, \ldots, t_{n-p}^{p-1}\right) v_{n+p}\right)\right]} \\
& \quad=O\left(\left(t_{2-p}^{p-1}, \ldots, t_{n-p}^{p-1}\right) v_{n+p+1}\right) .
\end{aligned}
$$

Let us explain the third relation. The action $v_{n+p-1}\left(t_{j}\right)$ for some $v_{m}$ inside $O\left(v_{n+1}\right)$ can appear only for $m \geq j+p \geq n+2 p-1$. On the other hand, by Lemma 5.1 $\left[v_{n+p-1}, v_{n+1}\right]=O\left(v_{n+p+1}\right)$. The second and forth equations are obtained by similar arguments. Thus,

$$
\left[\tilde{v}_{n}, \tilde{v}_{n+p-1}\right]=-t_{n}^{p-2} v_{n+p}+t_{n-p+1}^{p-1} O\left(v_{n+p+1}\right)+O\left(\left(t_{2-p}^{p-1}, \ldots, t_{n-p}^{p-1}\right) v_{n+p}\right) .
$$

We repeat this process and observe that our additional factors cannot disappear:

$$
\left(\operatorname{ad} \tilde{v}_{n}\right)^{p-1}\left(\tilde{v}_{n+p-1}\right)=-v_{n+p}+t_{n-p+1}^{p-1} O\left(v_{n+p+1}\right)+O\left(\left(t_{2-p}^{p-1}, \ldots, t_{n-p}^{p-1}\right) v_{n+p}\right) .
$$

The last term can contain a summand with $v_{n+p}$, it is of the form $t_{i}^{p-1} r v_{n+p}$, where $r \in R$ and $2-p \leq i \leq n-p$. But, by construction, the element (15) is homogeneous. Then

$$
\begin{aligned}
\mathrm{wt}_{0}\left(v_{n+p}\right) & =\mathrm{wt}_{0}\left(t_{i}^{p-1} r v_{n+p}\right) \\
& =\mathrm{wt}_{0}\left(v_{n+p}\right)+\mathrm{wt}_{0}\left(t_{i}^{p-1} r\right) \\
& \leq \mathrm{wt}_{0}\left(v_{n+p}\right)-(p-1) \lambda_{0}^{i},
\end{aligned}
$$

a contradiction. Therefore, the last term (15) contains only $v_{m}$ with $m \geq n+p+1$. Then we set

$$
\tilde{v}_{n+p}=-\left(\operatorname{ad} \tilde{v}_{n}\right)^{p-1}\left(\tilde{v}_{n+p-1}\right)=v_{n+p}+O\left(\left(t_{2-p}^{p-1}, \ldots, t_{n-p+1}^{p-1}\right) v_{n+p+1}\right),
$$

and the induction step is proved.

Corollary 8.7. The Lie algebra $\boldsymbol{L}=\operatorname{Lie}_{p}\left(v_{1}, \ldots, v_{p}\right)$ is infinite-dimensional. 


\section{References}

[1] Y. A. Bahturin, A. A. Mikhalev, V. M. Petrogradsky, and M. V. Zaicev, Infinite-dimensional Lie superalgebras. De Gruyter Exp. Math. 7, Walter de Gruyter, Berlin 1992. Zbl 0762.17001 MR 1192546

[2] L. Bartholdi, Branch rings, thinned rings, tree enveloping rings. Israel J. Math. 154 (2006), 93-139. Zbl 1173.16303 MR 2254535

[3] L. Bartholdi, personal communication.

[4] W. Borho and H. Kraft, Über die Gelfand-Kirillov-Dimension. Math. Ann. 220 (1976), 1-24. Zbl 0306.17005 MR 0412240

[5] V. Drensky and L. Hammoudi, Combinatorics of words and semigroup algebras which are sums of locally nilpotent subalgebras. Canad. Math. Bull. 47 (2004), 343-353. Zbl 1069.16020 MR 2072594

[6] I. M. Gelfand and A. A. Kirillov, Sur les corps liés aux algèbres enveloppantes des algèbres de Lie. Inst. Hautes Études Sci. Publ. Math. 31 (1966), 5-19. Zbl 0144.02104 MR 0207918

[7] R. I. Grigorchuk, On Burnside's problem on periodic groups. Funktsional. Anal. $i$. Prilozhen. 14 (1980), 53-54; English transl. Funct. Anal. Appl. 14 (1980), 41-43. Zbl 0595.20029 MR 0565099

[8] R. I. Grigorchuk, Just infinite branch groups. In New horizons in pro-p groups, Progr. Math. 184, Birkhäuser, Boston 2000, 121-179. Zbl 0982.20024 MR 1765119

[9] R. I. Grigorchuk, Branch groups. Mat. Zametki 67 (2000), 852-858; English transl. Math. Notes 67 (2000), 718-723. Zbl 0994.20027 MR 1820639

[10] N. Gupta and S. Sidki, On the Burnside problem for periodic groups. Math. Z. 182 (1983), 385-388. Zbl 0513.20024 MR 696534

[11] G. H. Hardy and E. M. Wright, An introduction to the theory of numbers. 5th ed., Oxford University Press, Oxford 1979. Zbl 0423.10001 MR 0568909

[12] N. Jacobson, Lie algebras. Interscience Tracts Pure Appl. Math. 10, Interscience Publishers, New York 1962. Zbl 0121.27504 MR 0143793

[13] A. V. Kelarev, A sum of two locally nilpotent rings may be not nil. Arch. Math. (Basel) 60 (1993), 431-435. Zbl 0784.16011 MR 1213511

[14] G. R. Krause and T. H. Lenagan, Growth of algebras and Gelfand-Kirillov dimension. Grad. Stud. Math. 22, Amer. Math. Soc., Providence, RI, 2000. Zbl 0957.16001 MR 1721834

[15] Ya.S. Krylyuk, The enveloping algebra of Petrogradsky-Shestakov-Zelmanov algebra is not graded-nil in critical characteristics. J. Lie Theory, to appear.

[16] D. S. Passman and V. M. Petrogradsky, Polycyclic restricted Lie algebras. Comm. Algebra 29 (2001), 3829-3838. Zbl 1054.17014 MR 1857016

[17] V. M. Petrogradskii, On some types of intermediate growth in Lie algebras. Uspekhi Mat. Nauk 48 (1993), no. 5, 181-182; English transl. Russian Math. Surveys 48 (1993), no. 5, 181-182. Zbl 0823.17015 MR 1258773 
[18] V. M. Petrogradsky, Intermediate growth in Lie algebras and their enveloping algebras. J. Algebra 179 (1996), 459-482. Zbl 0964.17005 MR 1367858

[19] V. M. Petrogradsky, Examples of self-iterating Lie algebras. J. Algebra 302 (2006), 881-886. Zbl 1109.17008 MR 2293788

[20] V. M. Petrogradsky, Y. P. Razmyslov, and E. O. Shishkin, Wreath products and KaluzhninKrasner embedding for Lie algebras. Proc. Amer. Math. Soc. 135 (2007), 625-636. Zbl 1173.17008 MR 2262857

[21] V. M. Petrogradsky and I. P. Shestakov, Examples of self-iterating Lie algebras, 2. J. Lie Theory 19 (2009), 697-724. Zbl 05674965 MR 2599000

[22] D. E. Radford, Divided power structures on Hopf algebras and embedding Lie algebras into special-derivation algebras. J. Algebra 98 (1986), 143-170. Zbl 0583.16007 MR 825139

[23] Y. P. Razmyslov, Identities of algebras and their representations. Transl. Math. Monographs 138, Amer. Math. Soc., Providence, RI, 1994. Zbl 0827.17001 MR 1291603

[24] I. P. Shestakov and E. Zelmanov, Some examples of nil Lie algebras. J. Eur. Math. Soc. (JEMS) 10 (2008), 391-398. Zbl 1144.17013 MR 2390328

[25] M. K. Smith, Universal enveloping algebras with subexponential but not polynomially bounded growth. Proc. Amer. Math. Soc. 60 (1976), 22-24. Zbl 0347.17005 MR 0419534

[26] H. Strade and R. Farnsteiner, Modular Lie algebras and their representations. Monographs Textbooks Pure Appl. Math. 116, Marcel Dekker, New York 1988. Zbl 0648.17003 MR 0929682

Received August 17, 2009; revised June 5, 2010

V. M. Petrogradsky, Faculty of Mathematics and Computer Science, Ulyanovsk State University, Lev Tolstoy 42, Ulyanovsk, 432970, Russia

E-mail: petrogradsky@ rambler.ru

I. P. Shestakov, Instituto de Mathemática e Estatística, Universidade de Saõ Paulo, Caixa postal 66281, CEP 05315-970, Saõ Paulo, Brazil

E-mail: shestak@ime.usp.br

E. Zelmanov, Department of Mathematics, University of California, San Diego, La Jolla, CA 92093-0112, U.S.A.

E-mail: ezelmano@math.ucsd.edu 